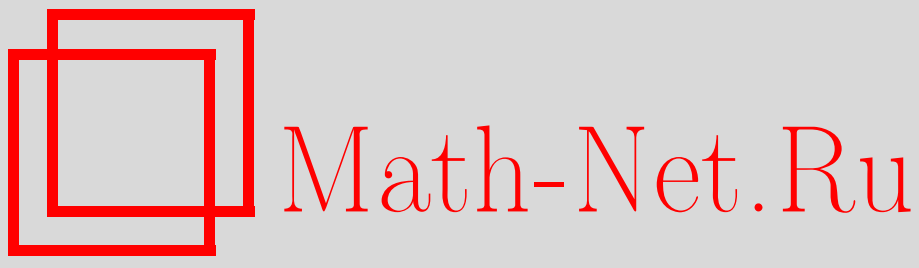

Е. А. Калита, Разрешимость нелинейных эллиптических систем в пространствах, слабее естественного энергетического, Изв. РАН. Сер. матем., 1997, том 61, выпуск $2,53-80$

DOI: https://doi.org/10.4213/im115

Использование Общероссийского математического портала Math-Net.Ru подразумевает, что вы прочитали и согласны с пользовательским соглашением

http: //www.mathnet.ru/rus/agreement

Параметры загрузки:

IP : 3.93 .64 .190

26 апреля 2023 г., 11:46:44 
УДК 517.956

\author{
Е. А. Калита
}

\title{
Разрешимость нелинейных эллиптических систем в пространствах, слабее естественного энергетического
}

\begin{abstract}
Вводится шкала пространств, двойственных классическим пространствам Морри. Для нелинейных эллиптических систем устанавливается разрешимость на некотором отрезке этой шкалы, величина отрезка существенно зависит от модуля эллиптичности системы. Как следствие, получена разрешимость системы для правой части из пространств Лебега с показателем, слабее соболевского, из пространства плотностей конечных борелевских мер, из пространств Харди c $p \leqslant 1$ при соответствующих явных условиях на модуль эллиптичности. Доказано существование и правильное поведение решений типа фундаментального решения. Результаты являются полностью новыми также для линейных систем с ограниченными разрывными коэффициентами.

Библиография: 19 наименований.
\end{abstract}

Разрешимость нелинейных эллиптических уравнений и систем произвольного порядка в естественном энергетическом пространстве хорошо известна. Основные результаты в этой области связаны с теорией операторов монотонного типа и были получены в 60-е годы (см. [1], [2] и приведенную там литературу). Отметим работу [3], где разрешимость рассматривалась для некоэрцитивного оператора, который образует коэрцитивную пару с некоторым вспомогательным оператором. Примерно тогда же в теории регулярности обобшенных решений был построен ряд контрпримеров [4]-[6], модифицировавшихся и обобшавшихся в дальнейшем многими авторами. Эти примеры показывают, что в пространстве, произвольно близком к естественному энергетическому (в шкалах Лебега или Морри, например), для нелинейной эллиптической системы, удовлетворяющей естественным структурным условиям, может не быть правильной зависимости решения от правой части. Позитивные результаты по разрешимости в пространствах, слабее естественного энергетического (при дополнительных ограничениях какого-либо рода), до сих пор практически отсутствуют. Для сравнения укажем, что нётеровость линейных эллиптических уравнений и систем с гладкими коэффициентами хорошо известна в достаточно широком наборе шкал пространств, например $W_{p}^{m}$ при $p \in(1, \infty)[7$, гл. 5].

В данной работе вводится новая шкала пространств, сопряженными к которым оказались пространства Морри. В $\S 1$ устанавливается разрешимость нелинейных эллиптических систем на некотором отрезке этой шкалы. Величина отрезка существенно зависит от модуля эллиптичности системы и в пределе занимает всю шкалу, когда модуль эллиптичности стремится к единице. В $\S 3$ получены явные оценки величины отрезка, точные в случае систем второго порядка. В работе рассматриваются системы из кордесовского класса [8]-[10], который обобшает классические уравнения типа Кордеса [11], с одной стороны, и дивергентные системы со

Работы выполнена при частичной поддержке Internat. Sci. Foundation (грант U97000).

$$
\text { (C) Е.А. КалитА } 1997
$$


стандартными структурными условиями - с другой. В $\S 2$ рассмотрено согласование решения, получаемого в $\S 1$, со стандартным обобщенным решением. Установлено, что если правая часть системы локально достаточно хорошая, то решение из дуального пространства Морри принадлежит в соответствующей подобласти основному энергетическому пространству.

Далее, возникает вопрос: насколько введенные пространства шире основного энергетического? В $\S 5$ для систем с правой частью из некоторых классических пространств, не вкладываюшихся в сопряженное к основному энергетическому, установлена разрешимость при соответствуюших ограничениях на модуль эллиптичности системы. Рассмотрены пространства Лебега с показателем, меньше соболевского, пространство плотностей борелевских мер, пространства Харди с $p \leqslant$ 1. Необходимые теоремы вложения получены в $\S 4$. В $\S 5$ при ограничениях на модуль эллиптичности также доказано существование и правильное поведение фундаментального решения для нелинейных эллиптических систем.

Идея о том, что свойства решений нелинейных и линейных с разрывными коэффищиентами эллиптических уравнений и систем могут сушественно зависеть от модуля эллиптичности, достаточно известна. В теории регулярности для квазилинейных недивергентных уравнений второго порядка Кордес предложил [11] интегральные оценки со степенным весом как методполучения довольно точной количественной зависимости гладкости решений от разброса собственных чисел матрицы коэффициентов. Для дивергентных систем произвольного порядка этот подход был развит в [12]. Отметим, что арифметическая часть метода Кордеса, связанная с разложением по сферическим функциям и с применением неравенства Харди, резко усложняется при попытках модификации канонического веса $r^{a}, r=\left|x-x_{0}\right|$. Распространение кордесовских оценок на бистепенной вес $\min \left\{r^{a} ; r^{b}\right\}$ позволило в [9], [10] исследовать поведение решений в изолированной особой точке.

Принципиальное развитие этого метода привело в данной работе к оценкам с ве$\operatorname{com}\left(\int|x-y|^{-a} d \sigma(y)\right)^{-1}, \sigma$-неотрицательная борелевская мера, $a>0$ зависит от модуля эллиптичности системы. Тем самым появилась возможность получения разрешимости в пространствах, которые слабее естественного энергетического не в окрестности одной точки, а во всем $\mathbb{R}^{n}$.

Отметим, что шкала пространства Морри и вводимая здесь шкала дуальных пространств Морри - единственные в настоящее время, в которых возможна количественная оценка величины интервала правильного поведения решений нелинейных эллиптических систем.

\section{§1. Разрешимость в дуальных пространствах Морри}

В пространстве $\mathbb{R}^{n}, n \geqslant 2$, рассматривается нелинейная эллиптическая системa

$$
\mathscr{L} u \equiv \operatorname{div}^{t} A\left(x, D^{s} u\right)=f(x)
$$

где $s+t=2 m, s, t$ целые неотрицательные, $m$ целое положительное, $u, f-$ вектор-функции размерности $N \geqslant 1, A$ - размерности $n^{t} N$. Предполагаем, что $A$ измерима, $A(x, 0)=0$ и выполнено следуюшее структурное условие: функция $B$, определяемая равенством

$$
\Delta^{m} u+\operatorname{div}^{t} B\left(x, D^{s} u\right)=\operatorname{div}^{t} A\left(x, D^{s} u\right)
$$

липшицева по последнему аргументу с константой меньше единищы:

$$
|B(x, \xi)-B(x, \eta)| \leqslant K|\xi-\eta|, \quad K<1 .
$$


Здесь $B$ имеет размерность $n^{t} N, D^{s} u$ (и соответственно $\xi, \eta$ )- размерность $n^{s} N$, так что

$$
\begin{aligned}
|B| & =\left[\sum_{i=1}^{N} \sum_{j_{1}=1}^{n} \cdots \sum_{j_{t}=1}^{n}\left(B_{j_{1} \ldots j_{t}}^{i}\right)^{2}\right]^{1 / 2}, \\
\left|D^{s} u\right| & =\left[\sum_{i=1}^{N} \sum_{j_{1}=1}^{n} \cdots \sum_{j_{s}=1}^{n}\left(D_{j_{1}} \ldots D_{j_{s}} u^{i}\right)^{2}\right]^{1 / 2},
\end{aligned}
$$

$D_{j}=d / d x_{j}$. Ради простоты условия (2) (чтобы не вводить полиномиальные коэффициенты) будем игнорировать факт совпадения смешанных производных при перемене порядка дифференцирования.

Остановимся подробнее на условии (2). В случае $s=2 m, t=0, N=1$ для уравнения

$$
\sum_{|\alpha|=2 m} A_{\alpha}(x) D^{\alpha} u=f(x),
$$

$A_{\alpha} \in L_{\infty}$, показатель $K$ легко выражается через коэффициенты (рассматриваем уравнения, эквивалентные с точностью до нормирующего множителя). Считаем мультииндексы упорядоченными в смысле (3), т.е. $\alpha=\left(j_{1}, \ldots, j_{|\alpha|}\right), j_{i} \in$ $\{1, \ldots, n\}$. Тогда

$$
\begin{aligned}
K & =\sup _{x} \min _{\varkappa \in \mathbb{R}} \max _{|\eta|=1} \sum_{|\alpha|,|\beta|=m}\left(\delta_{\alpha \beta}-\varkappa A_{\alpha \beta}(x)\right) \eta_{\alpha \beta} \\
& =\sup _{x} \min _{\varkappa}\left[\sum_{|\alpha|,|\beta|=m}\left(\delta_{\alpha \beta}-\varkappa A_{\alpha \beta}\right)^{2}\right]^{1 / 2} \\
& =\sup _{x} \min _{\varkappa}\left(n^{m}-2 \varkappa \sum_{|\alpha|=m} A_{\alpha \alpha}+\varkappa^{2} \sum_{|\alpha|=2 m} A_{\alpha}^{2}\right)^{1 / 2} \\
& =\sup _{x}\left(n^{m}-\left(\sum_{|\alpha|=m} A_{\alpha \alpha}\right)^{2} / \sum_{|\alpha|=2 m} A_{\alpha}^{2}\right)^{1 / 2}
\end{aligned}
$$

где $\varkappa$ - нормирующий множитель, $\delta_{\alpha \beta}$ - символ Кронекера. Таким образом, для уравнения (4) условие $K<1$ совпадает с классическим условием Кордеса для матрицы коэффициентов $\left(A_{\alpha}\right)$, рассматриваемой как квадратная матрица $n^{m} \times n^{m}$. Напомним, что условие Кордеса $K_{\varepsilon}[11]$ для матрищы $\left(a_{i j}\right), i, j=1, \ldots, M$, имеет вид

$$
\sum_{i} a_{i i} \geqslant\left((M-1+\varepsilon) \sum_{i, j} a_{i j}^{2}\right)^{1 / 2}, \quad \varepsilon>0 .
$$

Для дивергентных систем $(s=t=m, N \geqslant 1)$ условие (2) соответствует стандартной паре структурных условий

$$
\begin{aligned}
(A(x, \xi)-A(x, \eta))(\xi-\eta) & \geqslant d_{1}|\xi-\eta|^{2}, \\
|A(x, \xi)-A(x, \eta)| & \leqslant d_{2}|\xi-\eta|,
\end{aligned}
$$

причем $K^{2} \leqslant 1-d_{1}^{2} / d_{2}^{2}$. Действительно, поскольку $B(x, \xi)=A(x, \xi)-\xi$ при $s=t$, возводя (2) в квадрат, находим

$$
\left(1-K^{2}\right)|\xi-\eta|^{2}+|A(x, \xi)-A(x, \eta)|^{2} \leqslant 2(A(x, \xi)-A(x, \eta))(\xi-\eta),
$$


откуда с учетом $K<1$ следует первое условие (5). Второе следует из (2) по неравенству треугольника. В обратную сторону, если вьполнено (5), то

$$
\begin{aligned}
\mid B(x, \xi) & -\left.B(x, \eta)\right|^{2} \equiv|\xi-\eta-\varkappa A(x, \xi)+\varkappa A(x, \eta)|^{2} \\
& =|\xi-\eta|^{2}-2 \varkappa(A(x, \xi)-A(x, \eta))(\xi-\eta)+\varkappa^{2}|A(x, \xi)-A(x, \eta)|^{2} \\
& \leqslant\left(1-2 \varkappa d_{1}+\varkappa^{2} d_{2}^{2}\right)|\xi-\eta|^{2} .
\end{aligned}
$$

Выбирая нормирующий множитель $\varkappa=d_{1} / d_{2}^{2}$, получаем $(2)$ с $K=\left(1-d_{1}^{2} / d_{2}^{2}\right)^{1 / 2}$. Для дивергентных систем точное выражение $K$ через собственные числа матрицы $\partial A(x, \xi) / \partial \xi$, рассматриваемой как квадратная матрица $\left(n^{m} N\right) \times\left(n^{m} N\right)$, получено в $[12$, с. 33$]$.

Введем дуальные пространства Морри. Обозначим $L_{2, \omega}$ весовое пространство с нормой

$$
\|f\|_{\omega}^{2}=\int_{\mathbb{R}^{n}}|f|^{2} \omega d x
$$

и рассмотрим весовые функции вида

$$
\omega(x)=\left[\int_{\mathbb{R}^{n}}\left(|x-y|^{-a}+|x-y|^{-b}\right) d \sigma(y)\right]^{-1},
$$

где $\sigma$ - неотрицательная борелевская мера в $\mathbb{R}^{n}, \sigma\left(\mathbb{R}^{n}\right)=1$. При $0 \leqslant a<n$, $-n<b \leqslant a$ определим пространство $L_{2, a, b}$ с квазинормой

$$
\left\|f ; L_{2, a, b}\right\|=\inf _{\sigma}\|f\|_{\omega},
$$

вес $\omega$ задается формулой (6). При таком определении неравенство треугольника выполнено с константой больше единицы.

Легко видеть, что $L_{2, a, b}$ замкнуто относительно сложения: если $\omega_{1}, \omega_{2}$ - веса вида (6), определяемые мерами $\sigma_{1}, \sigma_{2}$, то для веса $\omega$, определяемого мерой $\sigma=$ $\frac{1}{2}\left(\sigma_{1}+\sigma_{2}\right)$, выполнено $\min \left\{\omega_{1} ; \omega_{2}\right\} \geqslant \omega / 2$, и, следовательно, $L_{2, \omega_{1}}+L_{2, \omega_{2}} \subset L_{2, \omega} \subset$ $L_{2, a, b}$.

Отметим очевидное вложение $L_{2, a, b} \subset L_{2, \alpha, \beta}, \alpha \geqslant a, \beta \leqslant b$.

ЗАмечАние 1 . Сопряженным к $L_{2, a, b}$ является один из вариантов классических пространств Морри - пространство $L_{2,-a,-b}$ с нормой

$$
\left\|g ; L_{2,-a,-b}\right\|^{2}=\sup _{y} \int_{\mathbb{R}^{n}}|g(x)|^{2}\left(|x-y|^{-a}+|x-y|^{-b}\right) d x .
$$

Действительно, если $f \in L_{2, a, b}, g \in L_{2,-a,-b}, 0 \leqslant a<n,-n<b \leqslant a$, то

$$
|(f, g)|=\left|\int f g d x\right| \leqslant \inf _{\sigma}\left(\|f\|_{\omega}\|g\|_{1 / \omega}\right) \leqslant\left\|f ; L_{2, a, b}\right\| \sup _{\sigma}\|g\|_{1 / \omega},
$$

и по теореме Фубини

$$
\begin{aligned}
\|g\|_{1 / \omega}^{2} & =\int|g(x)|^{2}\left[\int\left(|x-y|^{-a}+|x-y|^{-b}\right) d \sigma(y)\right] d x \\
& =\int\left[\int|g|^{2}\left(|x-y|^{-a}+|x-y|^{-b}\right) d x\right] d \sigma(y) \\
& \leqslant \sup _{y} \int|g|^{2}\left(|x-y|^{-a}+|x-y|^{-b}\right) d x \int d \sigma=\left\|g ; L_{2,-a,-b}\right\|^{2} .
\end{aligned}
$$


Тем самым $L_{2,-a,-b} \subset\left(L_{2, a, b}\right)^{*}$, причем $\|\mathrm{Id}\| \leqslant 1$ (здесь мы обозначаем через Id оператор вложения).

В обратную сторону, поскольку для каждого веса $\omega$ вида (6) $L_{2, \omega} \subset L_{2, a, b}$, $\|\operatorname{Id}\| \leqslant 1$, имеем $\left(L_{2, a, b}\right)^{*} \subset\left(L_{2, \omega}\right)^{*}=L_{2,1 / \omega},\|\operatorname{Id}\| \leqslant 1$. Выбирая в качестве плотности меры $\sigma$ дельта-функцию с носителем в точке $y$, получаем

$$
\int|g(x)|^{2}\left(|x-y|^{-a}+|x-y|^{-b}\right) d x \leqslant\left\|g ;\left(L_{2, a, b}\right)^{*}\right\|^{2} .
$$

В силу произвольности $y \in \mathbb{R}^{n}$ это дает $\left(L_{2, a, b}\right)^{*} \subset L_{2,-a,-b}$, причем $\|\mathrm{Id}\| \leqslant 1$.

Обозначим $H_{a, b}^{s}$ пространство с квазинормой

$$
\left\|u ; H_{a, b}^{s}\right\|=\left\|D^{s} u ; L_{2, a, b}\right\|
$$

(функции, различаюшиеся на полином степени меньше $s$, отождествляем), аналогично $H_{\omega}^{s}$ - пространство с нормой $\left\|u ; H_{\omega}^{s}\right\|=\left\|D^{s} u\right\|_{\omega}$. Также введем $H_{a, b}^{-t}-$ пространство с квазинормой

$$
\left\|f ; H_{a, b}^{-t}\right\|=\left\|I_{t} f ; L_{2, a, b}\right\|,
$$

где $I_{t}$ - оператор с символом $|\zeta|^{-t}$ (потенциал Рисса порядка $t$ ), аналогично $H_{\omega}^{-t}-$ пространство с нормой $\left\|f ; H_{\omega}^{-t}\right\|=\left\|I_{t} f\right\|_{\omega}$.

Рассмотрим оператор $T=T^{s, t}=D^{s} \Delta^{-m} \operatorname{div}^{t}, s+t=2 m$, который понимается как мультипликатор с символом

$$
\left(\zeta_{i_{1}} \ldots \zeta_{i_{s}}|\zeta|^{-2 m} \zeta_{j_{1}} \ldots \zeta_{j_{t}}\right), \quad i_{k}, j_{l}=1, \ldots, n
$$

и переводит вектор-функции размерности $n^{t}$ в вектор-функции размерности $n^{s}$. Обозначим через $T_{\omega}=T_{\omega}^{s, t}$ его норму в пространстве $L_{2, \omega}$ (строго говоря, из пространства $\left(L_{2, \omega}\right)^{n^{t}}$ в пространство $\left.\left(L_{2, \omega}\right)^{n^{s}}\right)$. Через $T_{a}=T_{a}^{s, t}$ обозначим соответствующую норму при $\omega(x)=|x|^{a}$. Поскольку $T$ - композиция проекторов Рисса, $T_{a}<\infty$ при $a \in(-n, n)$ [13]. Ниже, в $\S 3$, величина $T_{a}^{s, t}$ будет вычислена точно при $s+t=2$ и получена оценка $T_{a}^{s, t}$ в общем случае. Здесь мы используем только следующие свойства $T_{a}$, вытекающие из общей теории:

$-T_{a}^{s, t}$ логарифмически вьпукло по $a$ по интерполяционной теореме Стейна-Вейса (теорема Рисса-Торина для пространств Лебега с весом) [14, с. 156];

$-T_{0}^{s, t}=1$ по равенству Парсеваля;

$-T_{a}^{s, t} \rightarrow \infty$ при $|a| \rightarrow n-0$.

Последнее утверждение проверяется элементарно. Оператор $T$ является сверточным оператором в смысле главного значения с ядром вида $k(x)=r^{-n} \Omega(\theta)$, где $(r, \theta)$ - полярные координаты с полюсом в нуле, гладкая функция $\Omega$ удовлетворяет условию сокрашения $\int \Omega(\theta) d \theta=0$. Очевидно, найдутся точка $\theta_{0}$ на единичной сфере и число $\rho>0$ такие, что $k(x) \geqslant c>0$ при $\left|x-\theta_{0}\right|<\rho$ (буквой $c$ здесь и далее будем обозначать различные несущественные положительные константы). Пусть $f$ - характеристическая функция единичного шара. Тогда в области $\left\{x=(r, \theta):\left|\theta-\theta_{0}\right|<\rho / 2, \quad r>2 / \rho\right\}$ с коническим выходом на бесконечность имеем $T f(x) \geqslant c|x|^{-n}$, и, следовательно,

$$
\left\|T f ; L_{2}\left(\mathbb{R}^{n} ;|x|^{a}\right)\right\| \geqslant c(n-a)^{-1 / 2} \quad \text { при } \quad a \in(0, n) .
$$

Учитывая ограниченность $\left\|f ; L_{2}\left(\mathbb{R}^{n} ;|x|^{a}\right)\right\|$ при $a>0$, получаем $T_{a} \geqslant c(n-a)^{-1 / 2}$ при $a \rightarrow n-0$. По двойственности это дает также $T_{a} \geqslant c(n+a)^{-1 / 2}$ при $a \rightarrow-n+0$.

Определим числа $a^{*} \in(0, n), a_{*} \in(-n, 0)$ как корни уравнения $T_{a}^{s, t}=1 / K$, где $K$ - константа из условия (2). Указанные выше свойства $T_{a}$ гарантируют корректность этого определения. Явные оценки $a^{*}, a_{*}$ будут получены в $\S 3$, здесь отметим $a^{*} \rightarrow n, a_{*} \rightarrow-n$ при $K \rightarrow 0$. Отметим также $T_{a}^{s, t}<1 / K$ при $a \in\left(a_{*}, a^{*}\right)$. 
Tеорема 1. Пусть числа $a, b$ такие, что $a_{*}<b \leqslant 0 \leqslant a<a^{*}$. Тогда нелинейный оператор $\mathscr{L}: H_{a, b}^{s} \rightarrow H_{a, b}^{-t}$ однозначно обратим и $\mathscr{L}^{-1}$ липшич-непрерывен. Более того, для решения $u \in H_{a, b}^{s}$ системы (1) c $f \in H_{a, b}^{-t}$ при каждом весе $\omega$ вида (6) таком, что $f \in H_{\omega}^{-t}$, справедлива оценка

$$
c_{1}\left\|f ; H_{\omega}^{-t}\right\| \leqslant\left\|u ; H_{\omega}^{s}\right\| \leqslant c_{2}\left\|f ; H_{\omega}^{-t}\right\|,
$$

где константы $c_{1}, c_{2}$ зависят от $a, b, K, n, s, t$, но не от $\sigma, f$.

При доказательстве теоремы нам понадобится оценка $T_{\omega}^{s, t}$ для весов $\omega$ вида (6).

Лемма 1. Пусть $a, b \in(-n, n)$. Тогда для весов $\omega$ вида (6)

$$
T_{\omega}^{s, t} \leqslant \max \left\{T_{a}^{s, t} ; T_{b}^{s, t}\right\} .
$$

ДоКАЗАТЕЛЬСТво. Установим два простых свойства норм операторов в весовых пространствах.

1. Для произвольного веса $\omega$ и оператора $T$ в $L_{2, \omega}$ (вообще говоря, из одной декартовой степени $L_{2, \omega}$ в другую декартову степень $\left.L_{2, \omega}\right)$

$$
T_{\omega}=T_{1 / \omega}^{*} .
$$

Действительно, пусть $f \in L_{2, \omega}, g \in L_{2,1 / \omega},\|f\|_{\omega}=\|g\|_{1 / \omega}=1$. Тогда

$$
T_{\omega}=\sup _{f}\|T f\|_{\omega}=\sup _{f, g}(T f, g)=\sup _{f, g}\left(f, T^{*} g\right)=\sup _{g}\left\|T^{*} g\right\|_{1 / \omega}=T_{1 / \omega}^{*},
$$

где ( , ) обозначает скалярное спаривание элементов из сопряженных пространств.

2. Пусть вес $\omega$ имеет вид $\omega(x)=\int \omega_{\alpha}(x) d \sigma(\alpha)$, где множество параметров $\alpha$ считаем вложенным в $\mathbb{R}^{M}$ при некотором $M, \sigma$ - неотрицательная борелевская мера в $\mathbb{R}^{M}$, веса $\omega_{\alpha}$ такие, что $\bigcap_{\alpha} L_{2, \omega_{\alpha}}$ плотно в $L_{2, \omega}$. Тогда для оператора $T$, ограниченного в $L_{2, \omega_{\alpha}}$ при всех $\alpha$,

$$
T_{\omega} \leqslant \sup _{\alpha} T_{\omega_{\alpha}}
$$

Действительно, для $f \in \bigcap_{\alpha} L_{2, \omega_{\alpha}}$ по теореме Фубини

$$
\begin{aligned}
\|T f\|_{\omega}^{2} & =\int|T f|^{2} \int \omega_{\alpha} d \sigma(\alpha) d x=\iint|T f|^{2} \omega_{\alpha} d x d \sigma(\alpha) \\
& \leqslant \sup _{\alpha} T_{\omega_{\alpha}}^{2} \iint|f|^{2} \omega_{\alpha} d x d \sigma(\alpha)=\sup _{\alpha} T_{\omega_{\alpha}}^{2}\|f\|_{\omega}^{2} .
\end{aligned}
$$

Поскольку оценка установлена для плотного в $L_{2, \omega}$ множества, при замыкании получаем, что она справедлива для всех $f \in L_{2, \omega}$.

Теперь для веса $\omega$ вида (6) легко получаем оценку (8):

$$
T_{\omega}=T_{1 / \omega}^{*} \leqslant \max \left\{T_{-a}^{*} ; T_{-b}^{*}\right\}=\max \left\{T_{a} ; T_{b}\right\} .
$$

Относительно плотности пересечения, требуемого в свойстве 2 , отметим, что пространство $L_{\infty}^{0}$ ограниченных финитных функций плотно в возникающих здесь пространствах. 
ДОкАЗАТЕЛЬСТво ТЕОРемЫ 1. Наряду с системой (1) рассмотрим систему

$$
\mathscr{M} u \equiv(-\operatorname{div})^{s}\left(\omega T^{s, t} A\left(x, D^{s} u\right)\right)=(-\operatorname{div})^{s}\left(\omega D^{s} \Delta^{-m} f\right),
$$

где $\Delta^{-m}$ понимается как оператор с символом $\left(-|\zeta|^{2}\right)^{-m}$, вес $\omega$ вида (6) такой, что $f \in H_{\omega}^{-t}$. Нелинейный оператор $\mathscr{M}: H_{\omega}^{s} \rightarrow\left(H_{\omega}^{s}\right)^{*}$ понимается в смысле равенства

$$
(\mathscr{M} u, \varphi)=\int \omega T^{s, t} A\left(x, D^{s} u\right) D^{s} \varphi d x \quad \forall \varphi \in H_{\omega}^{s} .
$$

Покажем, что оператор $\mathscr{M}$ липшиц-непрерывен. По лемме 1 и в силу ограниченности проекторов Рисса в $L_{2}$ со степенным весом $|x|^{a}, a \in(-n, n)$ [13], оператор $T^{s, t}$ будет ограничен в $L_{2, \omega}$ при $a, b \in(-n, n)$. Поэтому для $u, v, \varphi \in H_{\omega}^{s}$

$$
\begin{aligned}
|(\mathscr{M} u-\mathscr{M} v, \varphi)| & \leqslant\left\|T^{s, t} A\left(x, D^{s} u\right)-T^{s, t} A\left(x, D^{s} v\right)\right\|_{\omega}\left\|D^{s} \varphi\right\|_{\omega} \\
& \leqslant c\left\|A\left(x, D^{s} u\right)-A\left(x, D^{s} v\right)\right\|_{\omega}\left\|D^{s} \varphi\right\|_{\omega} \leqslant c\left\|D^{s}(u-v)\right\|_{\omega}\left\|D^{s} \varphi\right\|_{\omega} .
\end{aligned}
$$

Покажем, что оператор $\mathscr{M}$ сильно монотонен, т.е.

$$
(\mathscr{M} u-\mathscr{M} v, u-v) \geqslant c\left\|u-v ; H_{\omega}^{s}\right\|^{2} \quad \forall u, v \in H_{\omega}^{s} .
$$

Вспоминая структурное условие (2), имеем

$$
\mathscr{M} u=(-\operatorname{div})^{s}\left(\omega D^{s} u\right)+(-\operatorname{div})^{s}\left(\omega T^{s, t} B\left(x, D^{s} u\right)\right),
$$

где $B$ липшицева по второму аргументу с константой $K$. Для $u, v \in H_{\omega}^{s}$ по неравенству Гёльдера

$$
\begin{aligned}
(\mathscr{M} u-\mathscr{M} v, u-v) \geqslant & \left\|D^{s}(u-v)\right\|_{\omega}^{2} \\
& -\left\|T^{s, t}\left(B\left(x, D^{s} u\right)-B\left(x, D^{s} v\right)\right)\right\|_{\omega}\left\|D^{s}(u-v)\right\|_{\omega} .
\end{aligned}
$$

Далее, по (2)

$$
\begin{aligned}
& \left\|T^{s, t}\left(B\left(x, D^{s} u\right)-B\left(x, D^{s} v\right)\right)\right\|_{\omega} \\
& \quad \leqslant T_{\omega}^{s, t}\left\|B\left(x, D^{s} u\right)-B\left(x, D^{s} v\right)\right\|_{\omega} \leqslant T_{\omega}^{s, t} K\left\|D^{s}(u-v)\right\|_{\omega},
\end{aligned}
$$

что дает

$$
(\mathscr{M} u-\mathscr{M} v, u-v) \geqslant\left(1-K T_{\omega}^{s, t}\right)\left\|D^{s}(u-v)\right\|_{\omega}^{2} .
$$

По лемме 1 с учетом условия теоремы $a, b \in\left(a_{*}, a^{*}\right)$ имеем $K T_{\omega}^{s, t}<1$, что влечет сильную монотонность $\mathscr{M}$.

По следствию 2.3 из [2, с. 97] сильно монотонный липшиц-непрерывный оператор однозначно обратим, и обратный также сильно монотонен и липшиц-непрерьвен. Поэтому система $\mathscr{M} u=g$ однозначно разрешима для любой функции $g \in\left(H_{\omega}^{s}\right)^{*}$, причем

$$
\left\|u ; H_{\omega}^{s}\right\| \asymp\left\|g ;\left(H_{\omega}^{s}\right)^{*}\right\| .
$$

Покажем, что для $g=(-\operatorname{div})^{s}\left(\omega D^{s} \Delta^{-m} f\right)$ выполнено

$$
\left\|g ;\left(H_{\omega}^{s}\right)^{*}\right\| \asymp\left\|f ; H_{\omega}^{-t}\right\| .
$$

Имеем

$$
\left\|g ;\left(H_{\omega}^{s}\right)^{*}\right\| \leqslant\left\|D^{s} \Delta^{-m} f\right\|_{\omega}=\left\|D^{s} I_{s} I_{t} f\right\|_{\omega},
$$


где $I_{s}$ - оператор с символом $|\zeta|^{-s}$. Оператор $D^{s} I_{s}$ является композицией проекторов Рисса, их ограниченность в $L_{2, \omega}$ следует из ограниченности в $L_{2}\left(\mathbb{R}^{n} ;|x|^{a}\right)$, $L_{2}\left(\mathbb{R}^{n} ;|x|^{b}\right)$ аналогично лемме 1 . Поэтому получаем

$$
\left\|g ;\left(H_{\omega}^{s}\right)^{*}\right\| \leqslant c\left\|f ; H_{\omega}^{-t}\right\|
$$

В обратную сторону,

$$
\left\|f ; H_{\omega}^{-t}\right\|=\left\|I_{s} \operatorname{div}^{s} D^{s} \Delta^{-m} f\right\|_{\omega} \leqslant c\left\|D^{s} \Delta^{-m} f\right\|_{\omega},
$$

поскольку оператор $I_{s} \operatorname{div}^{s}$ ограничен в $L_{2, \omega}$. Применяя функционал $g \in\left(H_{\omega}^{s}\right)^{*} \mathrm{k}$ функции $v=\Delta^{-m} f \in H_{\omega}^{s}$, получаем

$$
\left\|g ;\left(H_{\omega}^{s}\right)^{*}\right\| \geqslant(g, v)\left\|v ; H_{\omega}^{s}\right\|^{-1}=\left\|D^{s} \Delta^{-m} f\right\|_{\omega}
$$

что завершает доказательство (10).

Следовательно, решение (9) класса $H_{\omega}^{s}$ удовлетворяет (7).

Эквивалентность (10) доказывает также, что функция $u \in H_{\omega}^{s}$ является решением системы (1) тогда и только тогда, когда она является решением (9). Действительно, если $u \in H_{\omega}^{s}$, то функция $h=\mathscr{L} u-f \in H_{\omega}^{-t}$. В силу (10) $h=0$ в смысле $H_{\omega}^{-t}$ равносильно тому, что

$$
(-\operatorname{div})^{s}\left(\omega D^{s} \Delta^{-m} h\right) \equiv \mathscr{M} u-(-\operatorname{div})^{s}\left(\omega D^{s} \Delta^{-m} f\right)=0
$$

в смысле $\left(H_{\omega}^{s}\right)^{*}$.

Справедливость оценки (7) для произвольного веса $\omega$ такого, что $f \in H_{\omega}^{-t}$, вытекает из единственности решения в $H_{a, b}^{s}$, устанавливаемой ниже. Укажем, однако, что оценка (7) не связана с единственностью качественно. Она справедлива, например, при структурном условии

$$
|B(x, \xi)| \leqslant K(\xi), \quad K<1
$$

вместо условия (2). В этом случае она устанавливается аналогично результатам по регулярности из $\S 2$.

Липшиц-непрерывность оператора $\mathscr{L}^{-1}$ в $H_{a, b}^{-t}$ следует из единственности и оценки (7). Действительно, если $u, v \in H_{a, b}^{s}, \mathscr{L} u=f, \mathscr{L} v=g$, то функция $w=u-v$ является решением системы

$$
\operatorname{div}^{t} \tilde{A}\left(x, D^{s} w\right)=f-g
$$

где

$$
\tilde{A}(x, \xi) \equiv A\left(x, \xi+D^{s} v(x)\right)-A\left(x, D^{s} v(x)\right)
$$

удовлетворяет структурному условию $(2)$ и $\tilde{A}(x, 0)=0$. Следовательно,

$$
\left\|w ; H_{a, b}^{s}\right\| \leqslant c\left\|f-g ; H_{a, b}^{-t}\right\| .
$$

Перейдем к доказательству единственности. Отметим, что единственность имеет место также в предельном случае $a=a^{*}, b=a_{*}$.

Пусть $u, v \in H_{a, b}^{s}$ - два решения системы $(1), a=a^{*}, b=a_{*}$. Выберем вес

$$
\omega(x)=\left[1+\int\left(|x-y|^{-a}+|x-y|^{-b}\right) d \sigma(y)\right]^{-1}
$$


где $\sigma$ - такая конечная борелевская мера, что $u, v \in H_{\omega}^{s}$. Легко видеть, что такая мера, единая для $u, v$, существует: если $u \in H_{\omega_{1}}^{s}, v \in H_{\omega_{2}}^{s}$, веса $\omega_{1}, \omega_{2}$ определены по мерам $\sigma_{1}, \sigma_{2}$, то достаточно взять $\sigma=\sigma_{1}+\sigma_{2}$. Поскольку $w=u-v \in H_{\omega}^{s}$, имеем $\Delta^{-m} \operatorname{div}^{s}\left(\omega D^{s} w\right) \in H_{1 / \omega}^{t}$, и справедливо интегральное тождество

$$
\int\left(A\left(x, D^{s} u\right)-A\left(x, D^{s} v\right)\right) D^{t} \Delta^{-m} \operatorname{div}^{s}\left(\omega D^{s} w\right) d x=0
$$

По условию (2) отсюда следует

$$
\left\|D^{s} w\right\|_{\omega}^{2}-K\left\|D^{s} w\right\|_{\omega}\left\|T^{t, s}\left(\omega D^{s} w\right)\right\|_{1 / \omega} \leqslant 0
$$

Для оператора $T=T^{t, s}$ на произвольной функции $\varphi \in L_{2,1 / \omega}$ имеем

$$
\begin{aligned}
& \|T \varphi\|_{1 / \omega}^{2}=\int|T \varphi|^{2}\left[1+\int\left(|x-y|^{-a}+|x-y|^{-b}\right) d \sigma(y)\right] d x \\
& \quad \leqslant \int|\varphi|^{2} d x+T_{-a}^{2} \iint|\varphi|^{2}|x-y|^{-a} d x d \sigma(y)+T_{-b}^{2} \iint|\varphi|^{2}|x-y|^{-b} d x d \sigma(y) .
\end{aligned}
$$

Поскольку $\left(T^{t, s}\right)^{*}=T^{s, t}$, по лемме $1 T_{-a}^{t, s}=T_{a}^{s, t}=1 / K, T_{-b}^{t, s}=T_{b}^{s, t}=1 / K$ при $a=a^{*}, b=a_{*}$. Следовательно,

$$
\left\|T^{t, s} \varphi\right\|_{1 / \omega}^{2} \leqslant K^{-2}\left(\|\varphi\|_{1 / \omega}^{2}-\left(1-K^{2}\right)\left\|\varphi ; L_{2}\right\|^{2}\right) .
$$

Из (11) получаем

$$
\left\|D^{s} w\right\|_{\omega}^{2}-\left\|D^{s} w\right\|_{\omega}\left(\left\|D^{s} w\right\|_{\omega}^{2}-\left(1-K^{2}\right)\left\|\omega D^{s} w\right\|^{2}\right)^{1 / 2} \leqslant 0
$$

откуда следует

$$
\left\|\omega D^{s} w\right\|=0
$$

Так как вес $\omega$ почти всюду положителен, это означает, что $w$ - полином степени меньше $s$, т.е. $u=v$ в смысле $H_{a, b}^{s}$.

\section{§2. Регулярность решений}

Рассмотрим вопрос о повьшении регулярности решений системы (1) в шкале дуальных пространств Морри вплоть до совпадения со стандартным обобщенным решением из $H^{s} \equiv W_{2}^{s}$, если правая часть системы имеет локально соответствующую регулярность. Дальнейшее повышение регулярности обобщенных решений из $H^{s}$ в шкале классических пространств Морри достаточно исследовано (см. [8]-[12]).

Поскольку регулярность изучается локально, вместо $H_{a, b}^{s}$ используем пространства $H_{a}^{s}, a \in(0, n)$, определяемые аналогично по весовым функциям вида

$$
\omega(x)=\left(\int|x-y|^{-a} d \sigma(y)\right)^{-1}
$$

При исследовании регулярности структурные условия, налагаемые на систему (1), можно несколько ослабить (в сравнении с разрешимостью, здесь излишни 
монотонность и непрерывность). Вместо (2) здесь будем предполагать выполненHLIM

$$
|B(x, \xi)| \leqslant K(\xi), \quad K<1 .
$$

Очевидно, можно допустить зависимость $B$ от младших производных и рассматривать условие вида

$$
\left|B\left(x, \xi_{0}, \ldots, \xi_{s}\right)\right| \leqslant K\left|\xi_{s}\right|+c \sum_{j<s}\left|\xi_{j}\right|^{p_{j}}+g(x), \quad K<1
$$

где показатели $p_{j}$ выбраны так, чтобы обеспечить подчиненность младших членов главной части по теоремам вложения. Здесь это не делается ввиду стандартности подобного обобщения.

ТЕОРема 2. Пусть $\Omega$ - ограниченная область в $\mathbb{R}^{n}, \quad u \in H_{a}^{s}(\Omega)$ - решение системь (1) в $\Omega, a=a^{*}$. Тогда если $f \in H_{b}^{-t}(\Omega)$ для некоторого $b$, $0 \leqslant b<a^{*}$, mo $u \in H_{b, \mathrm{loc}}^{s}(\Omega)$.

ДокАЗАтЕльСтво. Пусть $\Omega_{1} \subset \subset \Omega-$ внутренняя подобласть, $\varphi \in \stackrel{0}{C}^{\infty}(\Omega)-$ срезающая функция: $\varphi=1$ в $\Omega_{1}, 0 \leqslant \varphi \leqslant 1$, а также $Q=\operatorname{supp} D \varphi \subset \subset$ $\Omega \backslash \Omega_{1}$. Пусть $\sigma$ - неотрицательная конечная борелевская мера в $\mathbb{R}^{n}$ такая, что $u \in H_{1 / \mu}^{s}(\Omega), f \in H_{1 / \nu}^{-t}(\Omega)$ для

$$
\mu(x)=\int|x-y|^{-a} d \sigma(y), \quad \nu(x)=\int|x-y|^{-b} d \sigma(y) .
$$

Сушествование такой меры $\sigma$, единой для $u$ и $f$, очевидно: если $\mu$ определено по мере $\sigma_{1}$, а $\nu$ - по $\sigma_{2}$, достаточно взять $\sigma=\sigma_{1}+\sigma_{2}$. Выберем вес $\omega=(\theta \mu+\nu)^{-1}$, $\theta>0$ - числовой параметр. Положим $v=\Delta^{-m} \operatorname{div}^{s}\left(\omega \varphi D^{s} u\right)$ и подставим в интегральное тождество пробную функцию $v \varphi$ :

$$
\int\left(A\left(x, D^{s} u\right) D^{t}(v \varphi)+f v \varphi\right) d x=0 .
$$

Как и при доказательстве леммы 1 , легко проверяется ограниченность оператора $T^{t, s}$ в $L_{2,1 / \omega}$, так что $v \in H_{1 / \omega}^{t}$, и такая подстановка допустима. Учитьвая условие (12), находим

$$
\begin{aligned}
\int A\left(x, D^{s} u\right) D^{t}(v \varphi) d x & =\int A\left(x, D^{s} u\right) \varphi D^{t} v d x+\int_{Q} \ldots \\
& =\int D^{s}(u \varphi) \omega \varphi D^{s} u d x+\int B \varphi D^{t} v d x+\int_{Q} \ldots \\
& \geqslant\left\|\varphi D^{s} u\right\|_{\omega}^{2}-K\left\|\varphi D^{s} u\right\|_{\omega}\left\|D^{t} v\right\|_{1 / \omega}+\int_{Q} \ldots
\end{aligned}
$$

где в многоточие включены члены, содержащие производные $\varphi$. Поскольку $D^{t} v=T^{t, s}\left(\omega \varphi D^{s} u\right)$, так же как при доказательстве леммы 1 , имеем

$$
\begin{aligned}
\left\|D^{t} v\right\|_{1 / \omega}^{2} & =\left\|D^{t} v\right\|_{\theta \mu}^{2}+\left\|D^{t} v\right\|_{\nu}^{2} \leqslant\left(T_{-a}^{t, s}\right)^{2}\left\|\omega \varphi D^{s} u\right\|_{\theta \mu}^{2}+\left(T_{-b}^{t, s}\right)^{2}\left\|\omega \varphi D^{s} u\right\|_{\nu}^{2} \\
& =\left(T_{a}^{s, t}\right)^{2}\left\{\left\|\varphi D^{s} u\right\|_{\omega}^{2}-\left[1-\left(T_{b}^{s, t} / T_{a}^{s, t}\right)^{2}\right]\left\|\omega \varphi D^{s} u\right\|_{\nu}^{2}\right\} .
\end{aligned}
$$


Поскольку $T_{a}^{s, t}=1 / K$ при $a=a^{*}, T_{b}^{s, t}<1 / K$ при $0 \leqslant b<a^{*}$, по элементарному неравенству $\sqrt{x(x-y)} \leqslant x-y / 2,0 \leqslant y \leqslant x$, получаем

$$
\left\|\varphi D^{s} u\right\|_{\omega}^{2}-K\left\|\varphi D^{s} u\right\|_{\omega}\left\|D^{t} v\right\|_{1 / \omega} \geqslant c\left\|\omega \varphi D^{s} u\right\|_{\nu}^{2}
$$

с некоторым $c>0$. Из (13) по (14) находим

$\left\|\omega \varphi D^{s} u\right\|_{\nu}^{2} \leqslant c\left|\int f v \varphi d x\right|+c \int_{Q}\left[\left|D^{s} u\right| \sum_{j<t}\left|D^{j} v\right|+\left(\left|D^{t} v\right|+\omega\left|D^{s} u\right|\right) \sum_{j<s}\left|D^{j} u\right|\right] d x$.

Оценим правую часть в полученном неравенстве. Для слагаемого с $f$ имеем

$$
\left|\int f v \varphi d x\right|=\left|\int D^{s} \Delta^{-m}(f \varphi) \omega \varphi D^{s} u d x\right| \leqslant\left\|D^{s} \Delta^{-m}(f \varphi)\right\|_{1 / \nu}\left\|\omega \varphi D^{s} u\right\|_{\nu} .
$$

Очевидно равенство $D^{s} \Delta^{-m}=D^{s} I_{s} I_{t}$, где $I_{j}$ - оператор с символом $|\zeta|^{-j}$. Аналогично лемме 1 проверяется, что проекторы Рисса, а значит, и оператор $D^{s} I_{s}$, ограничены в $L_{2,1 / \nu}$. Поэтому

$$
\left\|D^{s} \Delta^{-m}(f \varphi)\right\|_{1 / \nu} \leqslant c\left\|f \varphi ; H_{1 / \nu}^{-t}\right\|
$$

и по неравенству Юнга

$$
\left|\int f v \varphi d x\right| \leqslant \varepsilon\left\|\omega \varphi D^{s} u\right\|_{\nu}^{2}+c_{\varepsilon}\left\|f \varphi ; H_{1 / \nu}^{-t}\right\|^{2}
$$

$\varepsilon>0$ произвольно малое.

Для оценки в (15) интеграла по $Q$ используем ограниченность дробных интегралов в пространствах Лебега с весом Макенхаупта. А именно,

$$
\begin{gathered}
\left\|I_{j} g ; L_{q, \rho}\right\| \leqslant c\left\|g ; L_{p, \pi}\right\|, \\
1<p<n / j, \quad 1 / q=1 / p-j / n, \quad \pi=\rho^{p / q}, \quad \rho \in A_{q(1-j / n)},
\end{gathered}
$$

где $\pi, \rho$ - весовые функции, $L_{p, \pi}-$ пространство с нормой $\left\|g ; L_{p, \pi}\right\|=$ $\left\|\pi^{1 / p} g ; L_{p}\left(\mathbb{R}^{n}\right)\right\|, A_{r}-$ класс Макенхаупта [13]. В (15) по неравенству Гёльдера

$$
\int_{Q}\left|D^{s} u\right| \sum_{j<t}\left|D^{j} v\right| d x \leqslant\left\|D^{s} u\right\|_{1 / \mu, Q} \sum_{j<t}\left\|D^{j} v\right\|_{\mu, Q},
$$

где $\|\cdot\|_{\mu, Q}$ обозначает норму в $L_{2, \mu}(Q)$. Нас будут интересовать условия ограниченности интеграла по $Q$ при $\theta \rightarrow 0(\theta$ из определения $\omega)$, поэтому здесь достаточно оценить старшие из встречающихся производных $D^{t-1} v$. Оператор $D^{t-1} \Delta^{-m} \operatorname{div}^{s}$, очевидно, мажорируется оператором свертки с ядром $|x|^{1-n}$, т.е. оператором $I_{1}$ :

$$
\left|D^{t-1} v(x)\right| \leqslant c I_{1}\left(\left|\omega \varphi D^{s} u\right|\right)(x) .
$$

Известно, что принадлежность веса классу Макенхаупта $\mu \in A_{q}(1<q<\infty)$ равносильна ограниченности проекторов Рисса в пространстве $L_{q, \mu}[13]$. Аналогично лемме 1 для ограниченности проекторов Рисса в $L_{q, \mu}$ достаточно их ограниченности в $L_{q}\left(\mathbb{R}^{n} ;|x|^{-a}\right)$, что имеет место при $-n(q-1)<a<n$. Следовательно, $\mu \in A_{q} \forall q \in(1, \infty)$. По (17), (16) получаем

$$
\left\|D^{t-1} v\right\|_{\mu, Q} \leqslant\left\|D^{t-1} v\right\|_{\mu, \mathbb{R}^{n}} \leqslant c\left\|\mu^{1 / 2} \omega \varphi D^{s} u ; L_{p}\right\|,
$$


где $p=\frac{2 n}{n+2}$ (при $n \geqslant 3$ в в случае $n=2$ можно сначала оценить норму $D^{t-1} v$ в $L_{2, \mu}(Q)$ через норму в $L_{2+\varepsilon, \mu}(Q)$ по неравенству Гёльдера, а потом применять теорему вложения). По неравенству Гёльдера

$$
\left\|\mu^{1 / 2} \omega \varphi D^{s} u ; L_{p}\right\| \leqslant\left\|\omega \varphi D^{s} u\right\|_{\nu}\left(\int_{\Omega}\left(\frac{\mu}{\nu}\right)^{n / 2} d x\right)^{1 / n} .
$$

Последний множитель конечен при $a-b<2 \frac{n-a}{n-2}$ :

$$
\begin{aligned}
\mu(x)^{n / 2} & =\left(\int|x-y|^{-a} d \sigma(y)\right)^{n / 2} \\
& \leqslant\left(\int|x-y|^{-b} d \sigma\right)^{n / 2-1} \int|x-y|^{-b-(a-b) n / 2} d \sigma \\
\int_{\Omega}\left(\frac{\mu}{\nu}\right)^{n / 2} d x & \leqslant \int_{\Omega} \frac{1}{\nu} \int|x-y|^{-b-(a-b) n / 2} d \sigma(y) d x \\
& \leqslant c \int\left(\int_{\Omega}|x-y|^{-b-(a-b) n / 2} d x\right) d \sigma \leqslant c \int d \sigma
\end{aligned}
$$

где мы учли, что $\nu$ отделено от нуля в ограниченных областях, перемена порядка интегрирования допустима по теореме Фубини. Полученные оценки дают

$$
\int_{Q}\left|D^{s} u\right| \sum_{j<t}\left|D^{j} v\right| d x \leqslant c\left\|D^{s} u\right\|_{1 / \mu, Q}\left\|\omega \varphi D^{s} u\right\|_{\nu}
$$

Рассмотрим в (15) остальные члены, интегрируемые по $Q$. Из симметрии условия Макенхаупта следует равносильность $\mu \in A_{q}$ условию $\mu^{-q^{\prime} / q} \in A_{q^{\prime}}, 1 / q^{\prime}+$ $1 / q=1$, поэтому $\mu^{1-q} \in A_{q}$ для всех $q \in(1, \infty)$. Оценка (16) вместе с интегральньм представлением Соболева стандартно дает теорему вложения, и с учетом $\mu^{1-q} \in A_{q}$ из $D^{s} u \in L_{2,1 / \mu, \text { lос }}(\Omega)$ следует конечность

$$
\left\|\mu^{-1 / 2} D^{s-1} u ; L_{q}(Q)\right\|, \quad q=\frac{2 n}{n-2}
$$

(пусть для краткости $n>2$ ). По неравенству Гёльдера находим

$$
\begin{aligned}
\int_{Q}\left(\left|D^{t} v\right|\right. & \left.+\omega\left|D^{s} u\right|\right) \sum_{j<s}\left|D^{j} u\right| d x \leqslant\left\|\mu^{-1 / 2} \sum_{j<s}\left|D^{j} u\right| ; L_{q}(Q)\right\| \\
& \times\left[\left\|D^{t} v\right\|_{\nu}\left(\int_{Q}\left(\frac{\mu}{\nu}\right)^{n / 2} d x\right)^{1 / n}+\left\|D^{s} u\right\|_{1 / \mu, Q}\left(\int_{Q}\left(\frac{\mu}{\nu}\right)^{n} d x\right)^{1 / n}\right] .
\end{aligned}
$$

Аналогично (18) интегралы от $\mu / \nu$ конечны при $a-b<\frac{n-a}{n-1}$. Оператор $T^{t, s}$, являюшийся композицией проекторов Рисса, ограничен в $L_{2, \nu}$ аналогично лемме 1 , поэтому

$$
\left\|D^{t} v\right\|_{\nu} \leqslant c\left\|\omega \varphi D^{s} u\right\|_{\nu}
$$

Применяя полученные оценки в (15), находим

$$
\left\|\omega \varphi D^{s} u\right\|_{\nu} \leqslant c\left(\left\|f \varphi ; H_{1 / \nu}^{-t}\right\|+\left\|D^{s} u ; L_{2,1 / \mu}(Q)\right\|+\sum_{j<s}\left\|\mu^{-1 / 2} D^{j} u ; L_{q}(Q)\right\|\right),
$$


если $b$ удовлетворяет условию $a-b<\frac{n-a}{n-1}$. Поскольку правая часть неравенства не зависит от $\theta$ (напомним, что $\omega=(\theta \mu+\nu)^{-1}$ ), при $\theta \rightarrow 0$ получаем $\left\|\varphi D^{s} u\right\|_{1 / \nu}<\infty$, так что $D^{s} u \in L_{2, b}\left(\Omega_{1}\right)$. Поскольку внутренняя подобласть $\Omega_{1}$ произвольна, имеем $u \in H_{b, \text { loc }}^{s}(\Omega)$.

Если показатель $b(f)$ из условия $f \in H_{b(f)}^{-t}$ не удовлетворяет неравенству $a-b<\frac{n-a}{n-1}$, модифицируем определение веса $\nu$. Положим $b=b_{1}$ в определении $\nu$, где $b_{1} \in\left(a-\frac{n-a}{n-1}, a\right)$ и $b_{1}$ достаточно близко к левому концу интервала. Тогда проведенные вьше рассуждения доказывают $u \in H_{b, \text { loc }}^{s}(\Omega)$ с $b=b_{1}$.

Положим $a=b_{1}$ и повторим проведенные рассуждения с $b=b_{2}$, где $b_{2}>a-\frac{n-a}{n-1}$ достаточно близко к $a-\frac{n-a}{n-1}$, а также $b_{2} \geqslant b(f)$. Поскольку ограничения на $a-b$ ослабляются с уменьшением $a$, за конечное число шагов получаем $u \in H_{b, \text { loc }}^{s}(\Omega)$ с $b=b(f)$.

\section{§3. Оценка нормы модельного оператора}

Пусть, как и выше, $T^{s, t}=D^{s} \Delta^{-m} \operatorname{div}^{t}(s, t, m$ - целые неотрицательные, $s+t=$ $2 m)$ - сингулярный интегральньй оператор в $\mathbb{R}^{n}$, переводящий вектор-функции размерности $n^{t}$ в вектор-функции размерности $n^{s}$. Пусть $T_{a}^{s, t}$ - его норма в пространстве $L_{2}\left(\mathbb{R}^{n} ;|x|^{a}\right)$ (строго говоря, из декартовой степени в декартову степень этого пространства). При $a \in(-n, n)$ определим функции

$$
\begin{aligned}
M_{s, t}(a) & = \begin{cases}M_{1}(a)^{t} M_{2}(a)^{m-t}, & s \geqslant t, \\
M_{1}(a)^{s} M_{2}(-a)^{m-s}, & s \leqslant t,\end{cases} \\
M_{1}(a) & =\max _{j=1,2, \ldots} M_{1}\left(a, \lambda_{j}\right), \quad M_{2}(a)=\sup _{j=0,1, \ldots} M_{2}\left(a, \lambda_{j}\right), \\
M_{1}(a, \lambda) & =1+a^{2}\left(\lambda^{1 / 2}+\lambda^{-1 / 2} \frac{(n-2)^{2}-a^{2}}{4}\right)^{-2}, \\
M_{2}(a, \lambda) & =1+a\left[\lambda(a+n-3)+(n-1)\left(\frac{a+n-4}{2}\right)^{2}\right] \\
\times & \times\left[\lambda+\frac{(n-2)^{2}-(a-2)^{2}}{4}\right]^{-2},
\end{aligned}
$$

$\lambda_{j}=j(j+n-2)$ - собственные числа оператора Лапласа-Бельтрамина единичной сфере в $\mathbb{R}^{n}$. Основной результат параграфа составляет следующее утверждение.

Теорема 3. Имеем $T_{a}^{s, t} \leqslant M_{s, t}(a)^{1 / 2}$ nрu $a \in(-n, n)$. В случае $s+t=2$ неравенство переходит в равенство, а именно $T_{a}^{1,1}=M_{1}(a)^{1 / 2}$, $T_{a}^{2,0}=T_{-a}^{0,2}=M_{2}(a)^{1 / 2}$.

Что касается функций $M_{1}(a), M_{2}(a)$, отметим, что они легко вычисляются при исследовании $M_{k}(a, \lambda)$ на максимум по $\lambda$ с помошью дифференцирования. Действительно, $M_{1}(a, \lambda)$ возрастает по $\lambda$ при $0<\lambda<\frac{(n-2)^{2}-a^{2}}{4}$, если этот интервал не пуст, и убывает по $\lambda$ при $\lambda>\max \left\{0 ; \frac{(n-2)^{2}-a^{2}}{4}\right\}$. Поэтому

$$
M_{1}(a)=\max \left\{M_{1}\left(a, \lambda_{j}\right) ; M_{1}\left(a, \lambda_{j+1}\right)\right\},
$$


где $j$ определяется условием $\lambda_{j} \leqslant \frac{(n-2)^{2}-a^{2}}{4}<\lambda_{j+1}$. Решая уравнение $M_{1}\left(a, \lambda_{j}\right)=$ $M_{1}\left(a, \lambda_{j+1}\right)$ относительно $a$, находим

$$
\begin{gathered}
M_{1}(a)=M_{1}\left(a, \lambda_{j}\right) \quad \text { при } \quad a_{j} \leqslant|a|<a_{j-1}, \\
a_{0}=n, \quad a_{j}=\left[\max \left\{0 ;(n-2)^{2}-4\left(\lambda_{j} \lambda_{j+1}\right)^{1 / 2}\right\}\right]^{1 / 2}, \quad j \geqslant 1 .
\end{gathered}
$$

Отметим еще

$$
M_{1}(a)=M_{1}\left(a, \lambda_{1}\right)=1+a^{2}(n-1)\left(\frac{4}{n^{2}-a^{2}}\right)^{2} \quad \forall a \in(-n, n), \quad n \leqslant 8,
$$

а также $a_{1}<n-4$ для $n>8$.

Для функции $M_{2}$ укажем

$$
\begin{gathered}
M_{2}(a)=M_{2}\left(a, \lambda_{0}\right)=1+4 a \frac{n-1}{(n-a)^{2}}, \quad 0 \leqslant a<n, \\
M_{2}(a)=1, \quad 3-n \leqslant a \leqslant 0, \\
M_{2}(a)=M_{2}\left(a, \lambda_{2}\right), \quad-n<a<-n+1+\sqrt{3}, \quad n \geqslant 3 ; \quad-n<a \leqslant 0, \quad n=2 .
\end{gathered}
$$

Также при $n \geqslant 3$ существует возрастающая последовательность $\left(a_{j}\right), j \geqslant 2$, $1+\sqrt{3}-n<a_{2}<a_{3}<\cdots, a_{j} \rightarrow 3-n$ при $j \rightarrow \infty$ и $M_{2}(a)=M_{2}\left(a, \lambda_{j}\right)$ для $a \in\left(a_{j-1}, a_{j}\right)$ (полагаем $\left.a_{1}=-n\right)$. В отличие от функции $M_{1}$ здесь $a_{j}$ не выражаются в радикалах, однако для $a \in(2-n, 3-n)$ имеем

$$
M_{2}(a)=\max \left\{M_{2}\left(a, \lambda_{j}\right) ; M_{2}\left(a, \lambda_{j+1}\right)\right\},
$$

где $j$ определено условием

$$
\lambda_{j} \leqslant-\frac{a+n-4}{2}\left(\frac{a+n-2}{2}-\frac{n-1}{a+n-3}\right)<\lambda_{j+1} .
$$

Функция $M_{2}(a)$ при $a \in(-n, 0)$ впервые появилась в работе Кордеса [11], для $a \in(0, n)$ она вычислена в [12, с. 29]. Величина $M_{1}(2-n)=1+\frac{(n-2)^{2}}{n-1}$ (без вычисления $M_{1}(a)$ для других $\left.a\right)$ есть в $\left[12\right.$, гл. 3]. Для произвольного $a M_{1}$ получена автором.

Главное приложение теоремы 3 в данной тематике - явная оценка интервала "хороших $a$ ", т.е. таких $a$, что $T_{a}^{s, t}<1 / K, K$ - константа из структурного условия (2). Функция $M_{s, t}(a)$ выпукла на $(-n, n), M_{s, t}(0)=1<1 / K^{2}, M_{s, t}(a) \rightarrow \infty$ при $a \rightarrow \pm n$, поэтому уравнение $M_{s, t}(b)=1 / K^{2}$ имеет по одному корню $b^{*} \in(0, n)$ и $b_{*} \in(-n, 0)$. Очевидно, $a^{*} \geqslant b^{*}, a_{*} \leqslant b_{*}$. Уравнение $M_{s, t}(b)=1 / K^{2}$ разрешимо в радикалах далеко не всегда, очевидные случаи разрешимости следуюшие:

1) $s=t=m$ - дивергентные системы произвольного порядка;

2) $t=0, s=2 m$ - недивергентные системы, в радикалах выражается положительный корень $b^{*}$;

3) $s=0, t=2 m-$ в радикалах выражается $b_{*}$.

Однако для проверки условия $a \in\left(b_{*}, b^{*}\right)$ нет необходимости находить $b^{*}, b_{*}$ : в силу монотонности $M_{s, t}$ на интервалах $(0, n),(-n, 0)$ справедливо следующее утверждение.

СлеДСТвИЕ 1. Пусть $a \in(-n, n)$ такое, что $M_{s, t}(a)<1 / K^{2}$. Тогда $a \in\left(a_{*}, a^{*}\right)$. 
ДокАЗАТЕЛЬСТВо ТЕОРЕМЫ 3. Докажем $T_{a}^{1,1}=M_{1}(a)^{1 / 2}$.

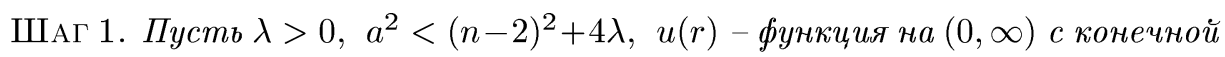
нормой

$$
|u|_{a}^{2}=\int_{0}^{\infty}\left({u^{\prime}}^{2}+\lambda r^{-2} u^{2}\right) r^{a+n-1} d r
$$

штрих обозначает дифференцирование по $r$. Тогда для решения на $(0, \infty)$ задачи

$$
\left(v^{\prime} r^{n-1}\right)^{\prime}-\lambda r^{n-3} v=\left(u^{\prime} r^{a+n-1}\right)^{\prime}-\lambda r^{a+n-3} u, \quad|v|_{-a}<\infty,
$$

справедлива оченка

$$
|v|_{-a}^{2} \leqslant M_{1}(a, \lambda)|u|_{a}^{2}
$$

ДокАЗАТЕЛЬСТво. Уравнение для $v$ является линейным уравнением Эйлера. Решая его методом неопределенных коэффициентов, получаем

$$
\begin{gathered}
v=u r^{a}-\frac{q_{2}}{\Delta} r^{q_{1}} v_{1}+\frac{q_{1}}{\Delta} r^{q_{2}} v_{2} \\
v_{1}(r)=a \int_{0}^{r} u_{1}(\rho) \rho^{a-1} d \rho, \quad v_{2}(r)=a \int_{+\infty}^{r} u_{2}(\rho) \rho^{a-1} d \rho,
\end{gathered}
$$

$u_{1}=u r^{-q_{1}}, u_{2}=u r^{-q_{2}}, q_{1}, q_{2}-$ корни характеристического уравнения $q^{2}+(n-2) q-\lambda=0, q_{1}<0<q_{2}, \Delta=q_{2}-q_{1}=\left((n-2)^{2}+4 \lambda\right)^{1 / 2}$. То, что $|v|_{-a}<\infty$, следует из $|u|_{a}<\infty$ по неравенству Харди

$$
\int_{0}^{\infty} z^{\prime 2} r^{\alpha+1} d r \geqslant \frac{\alpha^{2}}{4} \int_{0}^{\infty} z^{2} r^{\alpha-1} d r
$$

$\alpha$-произвольное вещественное число, функция $z$ удовлетворяет условию

$$
\liminf _{r \rightarrow \infty} z^{2}(r) r^{\alpha}=0 \text { при } \alpha>0, \quad \liminf _{r \rightarrow 0} z^{2}(r) r^{\alpha}=0 \text { при } \alpha<0 .
$$

Оценим норму $v$. Поскольку $v$ - решение задачи (23) и функция $u$ является допустимой пробной функцией в интегральном тождестве, имеем

$$
\begin{aligned}
|v|_{-a}^{2}-|u|_{a}^{2} & \equiv \int\left[\left(v^{\prime}-u^{\prime} r^{a}\right)\left(v^{\prime} r^{-a}+u^{\prime}\right)+\lambda r^{-2}\left(v-u r^{a}\right)\left(v r^{-a}+u\right)\right] r^{n-1} d r \\
& =\int\left[\left(v^{\prime}-u^{\prime} r^{a}\right)^{2}+\lambda r^{-2}\left(v-u r^{a}\right)^{2}\right] r^{n-1-a} d r
\end{aligned}
$$

С учетом формул Виета для корней характеристического многочлена имеем

$$
v^{\prime}=u^{\prime} r^{a}+\frac{\lambda}{\Delta}\left(r^{q_{1}-1} v_{1}-r^{q_{2}-1} v_{2}\right)
$$

что дает

$$
|v|_{-a}^{2}-|u|_{a}^{2}=\frac{\lambda}{\Delta} \int\left(q_{2} v_{1}^{2} r^{-\Delta}-q_{1} v_{2}^{2} r^{\Delta}\right) r^{-a-1} d r
$$


По неравенству Харди отсюда следует

$$
|v|_{-a}^{2}-|u|_{a}^{2} \leqslant \frac{\lambda}{\Delta} a^{2}\left[q_{2}\left(\frac{2}{\Delta+a}\right)^{2}-q_{1}\left(\frac{2}{\Delta-a}\right)^{2}\right] \int u^{2} r^{a+n-3} d r
$$

справедливость условия (26) для $v_{1}, v_{2}$ обеспечивается исходным предположением $|a|<\Delta$. Учитывая $2 q_{2}=\Delta+2-n,-2 q_{1}=\Delta+n-2$, после арифметических преобразований получаем

$$
|v|_{-a}^{2}-|u|_{a}^{2} \leqslant 4 \lambda a^{2}\left(\Delta^{2}-a^{2}\right)^{-2}\left(\Delta^{2}+a^{2}+2 a(n-2)\right) \int u^{2} r^{a+n-3} d r .
$$

С другой стороны, по неравенству Харди

$$
\begin{aligned}
|u|_{a}^{2} & \geqslant\left(\left(\frac{a+n-2}{2}\right)^{2}+\lambda\right) \int u^{2} r^{a+n-3} d r \\
& =\frac{1}{4}\left(\Delta^{2}+a^{2}+2 a(n-2)\right) \int u^{2} r^{a+n-3} d r
\end{aligned}
$$

что приводит к оценке (24):

$$
|v|_{-a}^{2}-|u|_{a}^{2} \leqslant 16 \lambda a^{2}\left(\Delta^{2}-a^{2}\right)^{-2}|u|_{a}^{2}=\left(M_{1}(a, \lambda)-1\right)|u|_{a}^{2} .
$$

ШАГ 2. Пусть $|a|<n, \quad u-$ функция в $\mathbb{R}^{n}$ с конечной нормой $\|D u\|_{a}=$ $\left\|D u ; L_{2}\left(\mathbb{R}^{n} ; r^{a}\right)\right\|, \quad r=|x|$. Тогда для решений уравнения

$$
\Delta v=\operatorname{div}\left(r^{a} D u\right)
$$

таких, что $\|D v\|_{-a}<\infty$, справедлива оченка

$$
\|D v\|_{-a}^{2} \leqslant M_{1}(a)\|D u\|_{a}^{2} .
$$

ДокАЗАТЕльСТво. Пусть $(r, \theta)$ - полярные координаты с полюсом в нуле. Разложим $u, v$ в ряд по полной ортонормированной системе сферических функций $Y_{j}(\theta), j=0,1, \ldots$, соответствуюших собственным числам $\lambda_{j}=j(j+n-2)$ оператора Лапласа-Бельтрами на единичной сфере в $\mathbb{R}^{n}$ (индекс, указывающий кратность $\lambda_{j}$, для краткости опускаем). Имеем

$$
u(x)=\sum_{j=0}^{\infty} u_{j}(r) Y_{j}(\theta), \quad u_{j}(r)=\int u(r, \theta) Y_{j}(\theta) d \theta,
$$

и аналогично для $v$. Для коэффициентов разложения $v_{j}$ уравнение $(28)$ переходит в (23) с $\lambda=\lambda_{j}, u=u_{j}$. Для норм имеем

$$
\begin{aligned}
\|D u\|_{a}^{2} & =\sum_{j=0}^{\infty} \int\left(u_{j}^{\prime 2}+\lambda_{j} r^{-2} u_{j}^{2}\right) r^{a+n-1} d r \equiv \sum_{j} U_{j}, \\
\|D v\|_{-a}^{2} & =\sum_{j=0}^{\infty} \int\left(v_{j}^{\prime 2}+\lambda_{j} r^{-2} v_{j}^{2}\right) r^{-a+n-1} d r \equiv \sum_{j} V_{j},
\end{aligned}
$$


и в силу шага 1

$$
V_{j} \leqslant M_{1}\left(a, \lambda_{j}\right) U_{j} \leqslant M_{1}(a) U_{j}, \quad j \geqslant 1,
$$

так как $(n-2)^{2}+4 \lambda_{j} \geqslant n^{2}>a^{2}$ при $j \geqslant 1$. Это также гарантирует сходимость ряда $\sum_{j} v_{j} Y_{j}$ в $L_{2, \text { loc }}\left(\mathbb{R}^{n} \backslash 0\right)$.

При $j=0$ решение порождаемого (28) уравнения имеет вид

$$
v_{0}(r)=\int^{r} u_{0}^{\prime}(\rho) \rho^{a} d \rho
$$

нижний предел интегрирования фиксируется произвольно. Тогда

$$
V_{0}=\int v_{0}^{\prime 2} r^{-a+n-1} d r=\int u_{0}^{2} r^{a+n-1} d r=U_{0} \leqslant M_{1}(a) U_{0},
$$

что завершает доказательство (29).

ШАГ 3. Справедлива оценка $T_{a}^{1,1} \leqslant M_{1}(a)^{1 / 2}$.

ДокАЗАТЕльСтво. Пусть $f=\left(f_{1}, \ldots, f_{n}\right)$ - ограниченная финитная функция (достаточно ограничиться такими функциями, поскольку они плотны в $L_{2}\left(\mathbb{R}^{n} ; r^{a}\right)$ ). Тогда определена функция $u=\Delta^{-1} \operatorname{div} f$, и в силу ограниченности проекторов Рисса $\|D u\|_{a}<\infty$. Пусть $R<\infty, B_{R}=\{x:|x|<R\}, \varphi \in \stackrel{0}{C}^{\infty}\left(B_{2 R}\right)$ - срезающая функция: $0 \leqslant \varphi \leqslant 1, \varphi=1$ в $B_{R},|D \varphi| \leqslant c / R$. Обозначим $Q=B_{2 R} \backslash B_{R}, u_{Q}{ }^{-}$ среднее значение $u$ по множеству $Q$. Пусть $v$ - решение $(28), v_{Q}$ - среднее значение $v$ по $Q$. Тогда

$$
\begin{aligned}
\int|D u|^{2} \varphi r^{a} d x & =\int D u D\left(\left(u-u_{Q}\right) \varphi\right) r^{a} d x+\cdots=\int D v D\left(\left(u-u_{Q}\right) \varphi\right) d x+\cdots \\
& =\int \varphi D v D u d x+\cdots=\int D\left(\left(v-v_{Q}\right) \varphi\right) D u d x+\cdots \\
& =\int D\left(\left(v-v_{Q}\right) \varphi\right) f d x+\cdots=\int f \varphi D v d x+\cdots
\end{aligned}
$$

где в многоточия включены члены, содержащие производную $\varphi$. По неравенству Гёльдера и неравенству Пуанкаре они оцениваются величиной

$$
R^{a}\left\|D u ; L_{2}(Q)\right\|^{2}+R^{-a}\left\|D v ; L_{2}(Q)\right\|^{2} .
$$

Это стремится к нулю при $R \rightarrow \infty$ по абсолютной непрерывности интеграла, так как $\|D u\|_{a},\|D v\|_{-a}<\infty$. Следовательно,

$$
\|D u\|_{a}^{2}=\int f D v d x .
$$

По неравенству Гёльдера и по шагу 2 получаем

$$
\|D u\|_{a}^{2} \leqslant\|D v\|_{-a}\|f\|_{a} \leqslant M_{1}(a)^{1 / 2}\|D u\|_{a}\|f\|_{a},
$$

откуда с учетом $D u=T^{1,1} f$ следует искомая оценка.

ШАГ 4. Справедлива оценка $T_{a}^{1,1} \geqslant M_{1}(a)^{1 / 2}$. 
ДокАЗАТЕЛЬСтво. Рассмотрим функцию

$$
u(x)=r^{\alpha} \varphi(r) Y_{j}(\theta),
$$

$\alpha=(2-n-a) / 2, j$ определяется условием $a_{j} \leqslant|a|<a_{j-1}, \varphi-$ срезающая функция: $0 \leqslant \varphi \leqslant 1, \varphi(r)=1$ при $1 / R<r<R, \varphi(r)=0$ при $r<1 /(2 R)$ или $r>2 R, r\left|\varphi^{\prime}(r)\right| \leqslant c$, где $R$ - произвольно большое положительное число. Решение соответствуюшего уравнения (28) имеет вид $v(r) Y_{j}(\theta)$, где $v$ дается формулой $(25)$ при $\lambda=\lambda_{j}=j(j+n-2), u(r)=r^{\alpha} \varphi$. Для функций $v_{1}, v_{2}$, интегрируя по частям, находим

$$
\begin{aligned}
v_{i}(r) & =\frac{a}{a+\alpha-q_{i}}\left(r^{a+\alpha-q_{i}} \varphi+w_{i}\right), \quad i=1,2, \\
\left|w_{1}(r)\right| & \leqslant c R^{q_{1}-\alpha-a} \chi(r-1 /(2 R))+c R^{a+\alpha-q_{1}} \chi(r-R), \\
\left|w_{2}(r)\right| & \leqslant c R^{a+\alpha-q_{2}} \chi(2 R-r)+c R^{q_{2}-\alpha-a} \chi(1 / R-r),
\end{aligned}
$$

где $\chi$ - характеристическая функция интервала $(0, \infty)$. Подставляя эти выражения в $(27)$, получаем

$$
\begin{aligned}
|v|_{-a}^{2}-|u|_{a}^{2}=\frac{\lambda a^{2}}{\Delta} & {\left[\frac{q_{2}}{\left(a+\alpha-q_{1}\right)^{2}} \int\left(\varphi+w_{1} r^{-(\Delta+a) / 2}\right)^{2} \frac{d r}{r}\right.} \\
& \left.-\frac{q_{1}}{\left(a+\alpha-q_{2}\right)^{2}} \int\left(\varphi+w_{2} r^{(\Delta-a) / 2}\right)^{2} \frac{d r}{r}\right] .
\end{aligned}
$$

Учитывая $|a|<\Delta$, легко видеть, что интегралы от слагаемых, содержащих $w_{1}, w_{2}$, оцениваются константами, не зависящими от $R$. Поскольку $a+\alpha-q_{1}=(a+\Delta) / 2$, $a+\alpha-q_{2}=(a-\Delta) / 2$, аналогично шагу 1 имеем

$$
|v|_{-a}^{2}-|u|_{a}^{2}=\lambda a^{2}\left(\Delta^{2}-a^{2}\right)^{-2}\left(\Delta^{2}+a^{2}+2 a(n-2)\right) \int \varphi^{2} \frac{d r}{r}+C,
$$

где $C$ - const. Подставляя $r^{\alpha} \varphi(r)$ в $(22)$, находим

$$
|u|_{a}^{2}=\left(\left(\frac{a+n-2}{2}\right)^{2}+\lambda\right) \int \varphi^{2} \frac{d r}{r}+C,
$$

где $C$ - const. При $R \rightarrow \infty$

$$
\int \varphi^{2} \frac{d r}{r} \geqslant \int_{1 / R}^{R} \frac{d r}{r}=2 \ln R \rightarrow \infty,
$$

поэтому для построенных функций

$$
|v|_{-a}^{2} /|u|_{a}^{2} \geqslant M_{1}\left(a, \lambda_{j}\right)-c / \ln R \rightarrow M_{1}(a) .
$$

Следовательно, для любого $\varepsilon>0$ найдется функция $u$ такая, что $\|D u\|_{a}<\infty$, и для решения уравнения (28) вьполнено

$$
\|D v\|_{-a}^{2} \geqslant\left(M_{1}(a)-\varepsilon\right)\|D u\|_{a}^{2} .
$$

Но для решения (28)

$$
\|D v\|_{-a}=\left\|T^{1,1}\left(r^{a} D u\right)\right\|_{-a} \leqslant T_{-a}^{1,1}\|D u\|_{a}=T_{a}^{1,1}\|D u\|_{a},
$$

и в силу произвольности $\varepsilon$ имеем $T_{a}^{1,1} \geqslant M_{1}(a)^{1 / 2}$.

Докажем $T_{a}^{2,0}=M_{2}(a)^{1 / 2}$ (равенство $T_{a}^{0,2}=T_{-a}^{2,0}$ следует из доказательства леммы 1). 
ШАГ 1. Пусть $|a|<n, \quad u-$ функиия в $\mathbb{R}^{n}$ с конечной нормой $\left\|D^{2} u\right\|_{a}=$ $\left\|D^{2} u ; L_{2}\left(\mathbb{R}^{n} ; r^{a}\right)\right\|, \quad r=|x|$. Тогда

$$
\left\|D^{2} u\right\|_{a}^{2} \leqslant M_{2}(a)\|\Delta u\|_{a}^{2}
$$

ДоКАЗАТЕЛЬСТвО. Интегрирование по частям дает

$$
\begin{aligned}
\left\|D^{2} u\right\|_{a}^{2}-\|\Delta u\|_{a}^{2} & =\int\left(-D_{j} u D_{i j}^{2} u D_{i} r^{a}+\Delta u D u D r^{a}\right) d x \\
& =\int\left(|D u|^{2} \Delta r^{a}-D_{i} u D_{j} u D_{i j}^{2} r^{a}\right) d x \\
& =a \int\left((n-1) u^{\prime 2}+(a+n-3) r^{-2} u_{\theta}^{2}\right) r^{a-2} d x,
\end{aligned}
$$

штрих обозначает дифференцирование по $r, u_{\theta}$ - градиент $u$ по угловым координатам, $(r, \theta)$ - система полярных координат с полюсом в нуле. Отсюда немедленно следует

$$
\left\|D^{2} u\right\|_{a} \leqslant\|\Delta u\|_{a}, \quad 3-n \leqslant a \leqslant 0 .
$$

Пусть $a \in(0, n)$. В сферических координатах $\Delta u=r^{1-n}\left(r^{n-1} u^{\prime}\right)^{\prime}+r^{-2} \Delta_{\theta} u$, где $\Delta_{\theta}$ - оператор Лапласа-Бельтрами на единичной сфере в $\mathbb{R}^{n}$, что дает

$$
\|\Delta u\|_{a}^{2}=\iint\left(\left(r^{n-1} u^{\prime}\right)^{\prime 2} r^{6-2 n}+2 r^{2} u_{\theta}^{\prime 2}+(2-a)(a+n-4) u_{\theta}^{2}+\left(\Delta_{\theta} u\right)^{2}\right) r^{a+n-5} d r d \theta .
$$

Поскольку первое отличное от нуля собственное число оператора $\Delta_{\theta}$ равно $n-1$, имеем

$$
\int\left(\Delta_{\theta} u(r, \theta)\right)^{2} d \theta \geqslant(n-1) \int\left(u_{\theta}(r, \theta)\right)^{2} d \theta \quad \forall r
$$

Применяя эту оценку и неравенство Харди, находим

$$
\|\Delta u\|_{a}^{2} \geqslant \int\left[\left(\frac{n-a}{2}\right)^{2} u^{\prime 2}+\left((a+n-4) \frac{n-a}{2}+n-1\right) u_{\theta}^{2} r^{-2}\right] r^{a-2} d x .
$$

Сравнивая соответствующие коэффициенты здесь и в (31), получаем (30) для положительных $a$, так как в этом случае

$$
\begin{aligned}
& \left(M_{2}(a)-1\right)\left((a+n-4) \frac{n-a}{2}+n-1\right)-a(a+n-3) \\
& \quad=a\left[4 \frac{n-1}{(n-a)^{2}}\left((a+n-4) \frac{n-a}{2}+n-1\right)-(a+n-4)-1\right] \\
& \quad=a\left(2 \frac{n-1}{n-a}-1\right)\left(a+n-4+2 \frac{n-1}{n-a}+1\right) \\
& \quad=a(n-2+2 a)(n-2+a)(n+1-a)(n-a)^{-2}>0 .
\end{aligned}
$$

Для получения оценки (30) при $a \in(-n, 3-n)(a \in(-n, 0)$ при $n=2)$ удобно разложить $u$ в ряд по гармоническим полиномам. Пусть $Y_{j}(\theta), j=0,1, \ldots,-$ 
полная ортонормированная система сферических функций, соответствуюших собственным числам $\lambda_{j}=j(j+n-2)$ оператора $\Delta_{\theta}$ (индекс, указывающий кратность $\lambda_{j}$, для краткости опускаем). Имеем

$$
u(x)=\sum_{j=0}^{\infty} u_{j}(r) r^{j} Y_{j}(\theta), \quad u_{j}(r) \equiv r^{-j} \int u(r, \theta) Y_{j}(\theta) d \theta
$$

и далее,

$$
\begin{aligned}
\left\|D^{2} u\right\|_{a}^{2}-\|\Delta u\|_{a}^{2} & =a \sum_{j=0}^{\infty} \int\left((n-1) u_{j}^{\prime 2}+j(j-1)(a-2) u_{j}^{2} r^{-2}\right) r^{2 j+a+n-3} d r \\
& \equiv \sum_{j} J_{j} \\
\|\Delta u\|_{a}^{2} & =\sum_{j=0}^{\infty} \int\left(r^{2 j+n-1} u_{j}^{\prime}\right)^{\prime 2} r^{1-n-2 j+a} d r \equiv \sum_{j} I_{j}
\end{aligned}
$$

Очевидно, $J_{j} \leqslant 0$ при $j=0,1$. При $j \geqslant 2$ по неравенству Харди

$$
\begin{aligned}
& J_{j} \leqslant\left(j(j-1) \frac{4 a(a-2)}{(2 j+a+n-4)^{2}}+a(n-1)\right) \int u_{j}^{\prime 2} r^{2 j+a+n-3} d r, \\
& I_{j} \geqslant\left(j+\frac{n-a}{2}\right)^{2} \int u_{j}^{\prime 2} r^{2 j+a+n-3} d r .
\end{aligned}
$$

Простые арифметические преобразования дают равенство

$$
\begin{aligned}
& a\left[(n-1)\left(j+\frac{a+n-4}{2}\right)^{2}+j(j-1)(a-2)\right] \\
& \quad \times\left(j+\frac{a+n-4}{2}\right)^{-2}\left(j+\frac{n-a}{2}\right)^{-2}=M_{2}\left(a, \lambda_{j}\right)-1,
\end{aligned}
$$

что завершает доказательство оценки (30).

ШАГ 2. Справедлива оценка $T_{a}^{2,0} \leqslant M_{2}(a)^{1 / 2}$.

ДокАЗАтЕльство. Пусть $f \in L_{\infty}$, т.е. $f$-ограниченная финитная функция. Тогда определена функция $u=\Delta^{-1} f$, и в силу ограниченности проекторов Рисса $\left\|D^{2} u\right\|_{a}<\infty, a \in(-n, n)$. Из (30) немедленно следует

$$
\left\|T^{2,0} f\right\|_{a}^{2} \leqslant M_{2}(a)\|f\|_{a}^{2}
$$

Учитывая плотность $L_{\infty}^{0}$ в $L_{2}\left(\mathbb{R}^{n} ; r^{a}\right)$, получаем искомую оценку нормы $T^{2,0}$ на всем пространстве $L_{2}\left(\mathbb{R}^{n} ; r^{a}\right)$.

ШАГ 3. Справедлива оценка $T_{a}^{2,0} \geqslant M_{2}(a)^{1 / 2}$. 
ДоКАЗАТЕЛЬСтво. Оценка устанавливается при рассмотрении функций вида $f=\Delta u, u=r^{\alpha} \varphi(r) Y_{j}(\theta), \alpha=\frac{4-n-a}{2}, j=0,1, \ldots$, вполне аналогично шагу 4 доказательства равенства $T_{a}^{1,1}=M_{1}(a)^{1 / 2}$.

Указанная в теореме оценка $T_{a}^{s, t}$ при $s+t=2 m>2$ следует из найденных оценок $T_{a, 1}^{1,1}, T_{a}^{2,0}, T_{a}^{0,2}$ по мультипликативному свойству нормы, поскольку оператор $T^{s, t}$ является тензорным произведением $\left(\otimes T^{2,0}\right)^{m-t}\left(\otimes T^{1,1}\right)^{t}$ при $t \leqslant s$, и $\left(\otimes T^{0,2}\right)^{m-s}\left(\otimes T^{1,1}\right)^{s}$ при $s \leqslant t$.

Укажем на точность оценки интервала "хороших $a$ " для систем второго порядка, получаемой при объединении теорем 1,3 . В теории регулярности соответствующие контрпримеры, являюшиеся тонкой модификацией контрпримера де Джорджи [4], построены в $\left[12\right.$, гл. 6] в случаях $s=t=1, a_{*}=2-n ; s=t=2, a_{*}=2-n$, $4-n$. Контрпримеры, построенные в [9], [10], [15] приводят к следующему результату.

УТВЕРЖДЕНИЕ. Пусть мы находимся в одной из следующих ситуаций:

1) $s=t=1, \quad K \in(0,1), \quad a \in(-n, n)$ nрu $n \leqslant 8, \quad 0<K \leqslant M_{1}\left(a_{1}\right)^{-1 / 2}$, $a_{1} \leqslant|a|<n$ при $n>8, a_{1}$ определено (20);

2) $s=2, \quad t=0, \quad K \in(0,1), a \in(0, n)$;

3) $s=2, \quad t=0,0<K \leqslant\left(\frac{n+1}{2 n-1}\right)^{1 / 2},-n<a \leqslant 2-n$.

Пусть также $M_{s, t}(a)=1 / K^{2}$.

Тогда найдется линейная система вида $(1)$ с коэффичиентами из $L_{\infty}$, удовлетворяющая условию (2) с указанным $K$ и имеющая при $f \equiv 0$ решение $u(x)$ makoe, ито

$$
\left|D^{s} u(x)\right|^{2} \asymp|x|^{-n-a} .
$$

В случаях 1) при $n=2$ и 2) утверждение справедливо также для одного уравнения второго порядка.

Отметим, что функция, удовлетворяюшая (32), принадлежит $H_{\alpha, \beta}^{s}$ при любых $\alpha>a, \beta<a$ : достаточно взять вес $\omega(x)=\left(|x|^{-\alpha}+|x|^{-\beta}\right)^{-1} \asymp \min \left\{|x|^{\alpha} ;|x|^{\beta}\right\}$.

\section{§4. Теоремы вложения}

Рассмотрим, какие пространства ограниченно отображаются потенциалом Рисса $I_{\alpha}$ в дуальные пространства Морри. При различных $\alpha$ это оказываются либо пространства $L_{p, a}, p>1$, либо пространства Харди $H_{p}, 0<p \leqslant 1$. Отметим, что возникаюший показатель $p$ меньше соответствуюшего показателя соболевского вложения: происходит как бы уменьшение эффективной размерности $\mathbb{R}^{n}$ в зависимости от $a$. Будет рассмотрено также действие $I_{\alpha}$ на пространстве плотностей конечных борелевских мер.

Распространим введенное выше определение $L_{2, a, b}$ на произвольные $p>1$. При $0 \leqslant a<n,-n<b \leqslant a$ обозначим $L_{p, a, b}$ пространство с квазинормой

$$
\begin{gathered}
\left\|f ; L_{p, a, b}\right\|=\inf _{\sigma}\left\|f ; L_{p, \omega}\right\|, \\
\omega(x)=\left(\int_{\mathbb{R}^{n}}\left(|x-a|^{-a}+|x-y|^{-b}\right) d \sigma(y)\right)^{1-p},
\end{gathered}
$$

где $L_{p, \omega}=L_{p}\left(\mathbb{R}^{n} ; \omega\right)$ - пространство Лебега с весом, inf берется по конечным неотрицательным борелевским мерам $\sigma, \sigma\left(\mathbb{R}^{n}\right)=1$. Обозначим $L_{p, a}=L_{p, a, a}$. Очевидно, $L_{p, a, b} \subset L_{p, \alpha, \beta}$ при $\alpha \geqslant a, \beta \leqslant b$. 
ЗАмечаниЕ 2. Сопряженным к $L_{p, a, b}$ является пространство Морри $L_{p^{\prime},-a,-b}$, $1 / p+1 / p^{\prime}=1$, с нормой

$$
\left\|g ; L_{p^{\prime},-a,-b}\right\|^{p^{\prime}}=\sup _{y} \int_{\mathbb{R}^{n}}|g(x)|^{p^{\prime}}\left(|x-y|^{-a}+|x-y|^{-b}\right) d x .
$$

Доказательство полностью аналогично доказательству замечания 1.

Далее мы практически не будем обрашать внимания на показатель $b$, характеризуюший поведение функции на бесконечности, по следуюшим соображениям. Исследование поведения решений нелинейных эллиптических систем в окрестности одной точки, хотя и встречает несомненные принщипиальные трудности, представляется все же гораздо более прозрачным, чем исследование на более массивных множествах (так, показатель $a$ отвечает за всю конечную часть $\mathbb{R}^{n}$ ).

При $\alpha>0$ обозначим $I_{\alpha}$ потенциал Рисса - оператор с символом $|\zeta|^{-\alpha}$. При $\alpha \neq n, n+2, n+4, \ldots$ он является оператором свертки с ядром $|x|^{\alpha-n}$ с точностью до числового множителя.

TeOpema 4. Пусть $0 \leqslant b \leqslant a<n, \quad 1<q<\infty, \quad 0<\alpha<(n-a) / q^{\prime}$, $1 / p=1 / q+\alpha /(n-a)$. Тогда для $f \in L_{p, a, b}$

$$
\left\|I_{\alpha} f ; L_{q, a, b}\right\| \leqslant c\left\|f ; L_{p, a, b}\right\| .
$$

Отметим, что для классических пространств Морри вложение с двойственными показателями получено в [16].

ДокАЗАТЕЛЬСТво. Используем идею [17], позволяющую легко сводить оценки потенциалов Рисса к оценкам максимальной функции. Разобьем область интегрирования в определении $I_{\alpha} f(x)$ на две части:

$$
I_{\alpha} f(x)=\left(\int_{|x-y| \leqslant \rho}+\int_{|x-y|>\rho}\right)|x-y|^{\alpha-n} f(y) d y \equiv J_{1}+J_{2},
$$

где $\rho$, зависящее от $x$, выберем ниже. Обозначим $Q_{i}=\left\{y: 2^{i-1} \rho<|x-y| \leqslant 2^{i} \rho\right\}$, $i$ - целое от $-\infty$ до $+\infty$. Пусть $\omega-$ вес вида (33) такой, что $f \in L_{p, \omega}$. Для $J_{2}$ по неравенству Гёльдера

$$
\begin{aligned}
\left|J_{2}\right| \leqslant & \sum_{i>0} \int_{Q_{i}}|x-y|^{\alpha-n}|f(y)| d y \\
\leqslant & \sum_{i>0}\left(2^{i-1} \rho\right)^{\alpha-\frac{n-a}{p}}\left(\int_{Q_{i}}|x-y|^{-a}|f(y)|^{p} \omega(y) d y\right)^{1 / p} \\
& \times\left(\int_{Q_{i}}|x-y|^{-n} \omega(y)^{\frac{-1}{p-1}} d y\right)^{1-1 / p} .
\end{aligned}
$$

Для последнего интеграла с учетом явного вида $\omega$ и теоремы Фубини

$$
\begin{aligned}
\int_{Q_{i}}|x-y|^{-n} \omega^{\frac{-1}{p-1}} d y & =\int_{\mathbb{R}^{n}} \int_{Q_{i}}|x-y|^{-n}\left(|y-z|^{-a}+|y-z|^{-b}\right) d y d \sigma(z) \\
& \leqslant c \int\left(|x-z|^{-a}+|x-z|^{-b}\right) d \sigma(z)=c \omega(x)^{\frac{-1}{p-1}}
\end{aligned}
$$


поскольку интеграл по $Q_{i}$ при $a, b<n$ оценивается через $\left(2^{i} \rho\right)^{-a}+\left(2^{i} \rho\right)^{-b}$ при $|x-z| \leqslant 2^{i} \rho$ и через $|x-z|^{-a}+|x-z|^{-b}$ при $|x-z|>2^{i} \rho$. Обозначая

$$
\varphi(x)=\int|x-y|^{-a}|f(y)|^{p} \omega(y) d y,
$$

имеем

$$
\left|J_{2}\right| \leqslant c \rho^{\alpha-\frac{n-a}{p}} \varphi(x)^{\frac{1}{p}} \omega(x)^{-\frac{1}{p}} .
$$

Для $J_{1}$ по неравенству Гёльдера

$$
\begin{aligned}
\left|J_{1}\right| \leqslant & \sum_{i \leqslant 0} \int_{Q_{i}}|x-y|^{\alpha-n}|f(y)| d y \\
\leqslant & \sum_{i \leqslant 0}\left(2^{i} \rho\right)^{\alpha}\left[\int_{Q_{i}}|x-y|^{-n}\left(|f(y)|^{p} \omega(y)\right)^{\theta} d y\right]^{\frac{1}{\theta p}} \\
& \times\left(\int_{Q_{i}}|x-y|^{-n} \omega(y)^{\frac{-\theta}{\theta p-1}} d y\right)^{1-\frac{1}{\theta p}}
\end{aligned}
$$

где $1 / p<\theta<1$. Первый интеграл оценим через максимальную функцию:

$$
\int_{Q_{i}}|x-y|^{-n}\left(|f|^{p} \omega\right)^{\theta} d y \leqslant c M\left(|f|^{\theta p} \omega^{\theta}\right)(x)
$$

где

$$
M g(x) \equiv \sup _{R} R^{-n} \int_{|x-y|<R}|g(y)| d y .
$$

Для второго интеграла с учетом явного вида $\omega$ по неравенству Минковского

$$
\begin{aligned}
& \left(\int_{Q_{i}}|x-y|^{-n} \omega^{\frac{-\theta}{\theta p-1}} d y\right)^{1-\frac{1}{\theta p}} \\
& \leqslant\left(\int_{\mathbb{R}^{n}}\left[\int_{Q_{i}}|x-y|^{-n}\left(|y-z|^{-a}+|y-z|^{-b}\right)^{\gamma} d y\right]^{1 / \gamma} d \sigma(z)\right)^{1-1 / p} \leqslant c \omega(x)^{-1 / p},
\end{aligned}
$$

если $\gamma=\theta \frac{p-1}{\theta p-1}$ столь близко к единище, что $\gamma a<n$. За счет выбора $\theta$, достаточно близкого к единице, это всегда достижимо. Следовательно,

$$
\left|J_{1}\right| \leqslant c \rho^{\alpha}\left[M\left(|f|^{\theta p} \omega^{\theta}\right)(x)\right]^{1 /(\theta p)} \omega(x)^{-1 / p} .
$$

Выбирая $\rho^{n-a}=\left(M\left(|f|^{\theta p} \omega^{\theta}\right)\right)^{-1 / \theta} \varphi$, получаем

$$
\left|I_{\alpha} f(x)\right| \leqslant c\left(M\left(|f|^{\theta p} \omega^{\theta}\right)\right)^{-\frac{1}{\theta q}} \varphi^{\frac{\alpha}{n-a}} \omega^{-\frac{1}{p}} .
$$

Положим

$$
\mu(x)=\int\left(r^{-a}+r^{-b}\right)\left[d \sigma(y)+\left\|f ; L_{p, \omega}\right\|^{-p}|f(y)|^{p} \omega(y) d y\right],
$$




$$
\begin{aligned}
& r=|x-y| . \text { Учитывая } \varphi(x) \leqslant \mu(x)\left\|f ; L_{p, \omega}\right\|^{p}, \omega(x)^{-1} \leqslant \mu(x)^{p-1}, \text { находим } \\
&\left\|I_{\alpha} f ; L_{q, a, b}\right\|^{q} \leqslant 2^{q-1} \int\left|I_{\alpha} f\right|^{q} \mu^{1-q} d x \\
& \leqslant c \int\left(M\left(|f|^{\theta p} \omega^{\theta}\right)\right)^{\frac{1}{\theta}} d x\left\|f ; L_{p, \omega}\right\|^{\frac{p q \alpha}{n-a}} \leqslant c\left\|f ; L_{p, \omega}\right\|^{q},
\end{aligned}
$$

где последнее неравенство следует из классической оценки максимальной функции в пространствах Лебега:

$$
\left\|M g ; L_{h}\right\| \leqslant c\left\|g ; L_{h}\right\|, \quad h \in(1, \infty) .
$$

Пространства Харди $H_{p}, 0<p \leqslant 1$, являются естественным продолжением пространств Лебега на случай $p \leqslant 1$. В настоящее время для них известен ряд эквивалентных определений, мы воспользуемся определением через атомарные разложения [18]. Функция $a(x)$ в $\mathbb{R}^{n}$ называется $(p, \infty, N)$-атомом, если:

1) ее носитель сосредоточен в некотором шаре $B\left(x_{0}, R\right)=\left\{x:\left|x-x_{0}\right|<R\right\}$;

2) $|a(x)| \leqslant R^{-n / p}$ для всех $x$;

3) $\int a(x) P(x) d x=0$ для любого полинома $P$ степени $\operatorname{deg} P<N$.

Пространство $H_{p}$ состоит из распределений вида $f=\sum_{j=1}^{\infty} c_{j} a_{j}$ таких, что $\sum_{j}\left|c_{j}\right|^{p}<\infty$, где $a_{j}-(p, \infty, N)$-атомы с $N>n / p-n, c_{j}$ - числовые коэффициенты. Величина

$$
\left\|f ; H_{p}\right\|^{p}=\inf \sum_{j}\left|c_{j}\right|^{p}
$$

определяет метрику в $H_{p}$, где inf берется по различным разложениям $f$ на атомы.

TeOpema 5. Пусть $0 \leqslant a<n, 1<q<\infty, \alpha \geqslant(n-a) / q^{\prime}, n / p^{\prime}=(n-a) / q^{\prime}-$ $\alpha$. Тогда для $f \in H_{p}$

$$
\left\|I_{\alpha} f ; L_{q, a}\right\| \leqslant c\left\|f ; H_{p}\right\| .
$$

Здесь $p^{\prime}$ - формально сопряженньй по Гёльдеру показатель: $1 / p+1 / p^{\prime}=1$. В данном случае $p \in(0,1], p^{\prime} \in[-\infty, 0)$. Вложение классических пространств Морри в пространства Гёльдера с двойственными теореме 5 показателями составляет хорошо известную лемму Морри (напомним, что $H_{p}^{*}=C^{\gamma}, \gamma=n / p-n$ не целое).

ДокАЗАТЕЛЬСТво. Пусть $a(x)-(p, \infty, N)$-атом с носителем в шаре $B\left(x_{0}, R\right)$. Тогда при $\alpha \neq n, n+2, \ldots$

$$
\left|I_{\alpha} a(x)\right| \leqslant \begin{cases}c R^{\alpha-n / p}, & r \leqslant R \\ c R^{N+n-n / p} r^{\alpha-n-N}, & r>R\end{cases}
$$

$r=\left|x-x_{0}\right|$. Пусть $f \in H_{p}, \sum_{j} c_{j} a_{j}$-разложение $f$ на $(p, \infty, N)$-атомы с $N>$ $n / p-n$, атомы $a_{j}$ имеют носители в шарах $B\left(x_{j}, R_{j}\right)$. Обозначим $r_{j}=\left|x-x_{j}\right|$. По неравенству Гёльдера

$$
\left|I_{\alpha} f(x)\right|^{q} \leqslant\left(\sum_{j}\left|c_{j}\right|\left|I_{\alpha} a_{j}\right|\right)^{q} \leqslant\left(\sum_{j}\left|c_{j}\right| r_{j}^{-a}\right)^{q-1} \sum_{j}\left|c_{j}\right| r_{j}^{a(q-1)}\left|I_{\alpha} a_{j}\right|^{q} .
$$

Функция

$$
\omega(x)=\left(\sum_{j}\left|c_{j}\right|\right)^{q-1}\left(\sum_{j}\left|c_{j}\right| r_{j}^{-a}\right)^{1-q}
$$


является допустимым весом для $L_{q, a}$, который определен по мере $\sigma$ с плотностью $\left(\sum_{j}\left|c_{j}\right|\right)^{-1} \sum_{j}\left|c_{j}\right| \delta\left(x-x_{j}\right)$, где $\delta$ - дельта-функция Дирака. Сходимость ряда $c_{j}$ следует из вогнутости степенной функции с $p \leqslant 1$ :

$$
\sum_{j}\left|c_{j}\right| \leqslant\left(\sum_{j}\left|c_{j}\right|^{p}\right)^{1 / p} \sim\left\|f ; H_{p}\right\|
$$

Поэтому получаем

$$
\left\|I_{\alpha} f ; L_{q, a}\right\|^{q} \leqslant\left\|I_{\alpha} f ; L_{q, \omega}\right\|^{q} \leqslant c\left(\sum_{j}\left|c_{j}\right|\right)^{q-1} \int_{\mathbb{R}^{n}} \sum_{j}\left|c_{j}\right| r_{j}^{a(q-1)}\left|I_{\alpha} a_{j}\right|^{q} d x .
$$

Учитывая (34) и условие $N>n / p-n$, для каждого атома $a_{j}$ находим

$$
\int_{\mathbb{R}^{n}} r_{j}^{a(q-1)}\left|I_{\alpha} a_{j}\right|^{q} d x \leqslant C, \quad C-\mathrm{const},
$$

что дает искомую оценку при $\alpha \neq n, n+2, \ldots$ В этих исключительных случаях утверждение теоремы может быть получено применением доказанной оценки к оператору $I_{\alpha-\varepsilon}(\varepsilon>0$ достаточно малое) и последуюшим применением теоремы 4 к оператору $I_{\varepsilon}$.

TeOpema 6. Пусть $0 \leqslant a<n, \quad-n<b<a, q>1, \quad \alpha>0, \quad b<n-\alpha q^{\prime}<a$, где $1 / q+1 / q^{\prime}=1$. Тогда для плотности $f=d \sigma / d x$ борелевской меры $\sigma c$ конечной вариацией $\operatorname{var} \sigma=\int_{\mathbb{R}^{n}}|d \sigma|$ выполнена оценка

$$
\left\|I_{\alpha} f ; L_{q, a, b}\right\| \leqslant c \operatorname{var} \sigma .
$$

Потенциал Рисса от плотности меры $\sigma$, не являющейся абсолютно непрерьвной, понимается в смысле

$$
I_{\alpha} f(x)=\int|x-y|^{\alpha-n} d \sigma(y)
$$

ДокАЗАТЕЛЬСтво. По неравенству Гёльдера

$$
\left|I_{\alpha} f(x)\right|^{q} \leqslant\left(\int\left(r^{-a}+r^{-b}\right)|d \sigma(y)|\right)^{q-1} \int\left(r^{-a}+r^{-b}\right)^{1-q} r^{(\alpha-n) q}|d \sigma(y)|
$$

$r=|x-y|$. Полагая

$$
\omega(x)=\left(\frac{1}{\operatorname{var} \sigma} \int\left(r^{-a}+r^{-b}\right)|d \sigma(y)|\right)^{1-q}
$$

находим

$$
\left\|I_{\alpha} f ; L_{q, a, b}\right\|^{q} \leqslant\left\|I_{\alpha} f ; L_{q, \omega}\right\|^{q} \leqslant(\operatorname{var} \sigma)^{q-1} \iint\left(r^{-a}+r^{-b}\right)^{1-q} r^{(\alpha-n) q}|d \sigma(y)| d x .
$$

После перемены порядка интегрирования по теореме Фубини имеем

$$
\int\left(r^{-a}+r^{-b}\right)^{1-q} r^{(\alpha-n) q} d x=C<\infty, \quad C-\text { const. }
$$

при $(a-n)(q-1)+\alpha q>0>(b-n)(q-1)+\alpha q$, что завершает доказательство. 


\section{§5. Разрешимость с правой частью из классических пространств}

Пусть правая часть (1) записана в виде $f=\sum_{j} \operatorname{div}^{t-j} f_{j}$, т.е. мы имеем систему

$$
\operatorname{div}^{t} A\left(x, D^{s} u\right)=\sum_{j=0}^{t} \operatorname{div}^{t-j} f_{j}(x) .
$$

Рассмотрим, при каких $f_{j}$ имеет место разрешимость. Известные результаты для нелинейных эллиптических задач высокого порядка, вытекающие из теории монотонных операторов, гарантируют разрешимость при $f_{j} \in L_{p_{j}}$ с $p_{j}=$ $2 n /(n+2 j)$ при $j<n / 2, p_{j}>1$ при $j=n / 2, p_{j}=1$ при $j>n / 2$ (см., например, $[19$, гл. 1]; в [19] рассматривался дивергентный случай и ограниченная область, для неограниченной области при $j \geqslant n / 2$ следует добавить очевидные условия убывания $f_{j}$ на бесконечности). Результаты $\S 1,3,4$ легко позволяют для любого $p_{j}>1$ (при $j>0$ ) получить явные ограничения на показатель $K$ из $(2)$, при которых система (35) разрешима с $f \in L_{p_{j}}$, а для $\frac{n}{n+j}<p_{j} \leqslant 1-$ с $f_{j}$ из пространств Харди $H_{p_{j}}$. А именно, справедливо следующее утверждение.

ТЕОРема 7. Пусть число $a \in(0, n)$ и показатель $K$ из (2) удовлетворяют условию

$$
M_{s, t}(a)<1 / K^{2},
$$

где $M_{s, t}$ определено (19). Пусть при $j<\frac{n-a}{2} \quad f_{j} \in L_{p_{j}, a, 0}, \quad p_{j}=2 \frac{n-a}{n-a+2 j}$; при $j \geqslant \frac{n-a}{2} \quad f_{j} \in H_{p_{j}}, \quad p_{j}=\frac{2 n}{n+a+2 j}$. Тогда система (35) имеет решение $u \in H_{a, 0}^{s}$, причем

$$
\left\|u ; H_{a, 0}^{s}\right\| \leqslant c \sum_{j=0}^{t} F_{j},
$$

где $F_{j}=\left\|f_{j} ; L_{p_{j}, a, 0}\right\|$ при $0 \leqslant j<(n-a) / 2 \quad(0 \leqslant j \leqslant t$ nрu $2 t<n-a)$, $F_{j}=\left\|f_{j} ; H_{p_{j}}\right\| n p u(n-a) / 2 \leqslant j \leqslant t$.

Напомним, что $L_{p}\left(\mathbb{R}^{n}\right) \subset L_{p, a, 0}, a \geqslant 0$. Отметим, что в формулировке теоремы можно заменить $H_{a, 0}^{s}$ на $H_{a}^{s}$ при замене $L_{p, a, 0}$ на $L_{p, a}$.

ДокАЗАТЕЛЬСТво. По следствию 1 условие (36) обеспечивает $a \in\left(0, a^{*}\right)$. По теореме 1 система (1) разрешима в $H_{a, 0}^{s}$ для любого $f \in H_{a, 0}^{-t}$ с оценкой (7). Для $f=\sum_{j} \operatorname{div}^{t-j} f_{j}$ имеем

$$
\left\|f ; H_{a, 0}^{-t}\right\| \leqslant \sum_{j}\left\|\operatorname{div}^{t-j} I_{t-j} I_{j} f_{j} ; L_{2, a, 0}\right\| \leqslant c \sum_{j}\left\|I_{j} f_{j} ; L_{2, a, 0}\right\|,
$$

поскольку операторы $\operatorname{div}^{t-j} I_{t-j}$, являясь композициями проекторов Рисса, ограничены в $L_{2, a, 0}$ аналогично лемме 1 . По теоремам 4,5

$$
\left\|I_{j} f_{j} ; L_{2, a, 0}\right\| \leqslant c F_{j},
$$

что завершает доказательство.

Отметим, что по теореме 1 решение единственно.

Рассмотрим случай, когда $f_{j}$ - плотность борелевской меры, не обязательно абсолютно непрерывной. 
Tеорема 8. Пусть $j \in\{1, \ldots, t\}, \quad j \leqslant n / 2, u$ для показателя $K$ из (2) выполнено

$$
M_{s, t}(n-2 j)<1 / K^{2} .
$$

Пусть $f_{j}=d \sigma / d x-$ плотность борелевской мерь $\sigma$ в $\mathbb{R}^{n}$ с конечной вариацией $\operatorname{var} \sigma=\int_{\mathbb{R}^{n}}|d \sigma|$. Тогда система (1) $c f=\operatorname{div}^{t-j} f_{j}$ имеет решение $u \in H_{a, b}^{s}, \quad a=n-2 j+\varepsilon, \quad b=n-2 j-\varepsilon, \quad \varepsilon>0$ достаточно малое, причем

$$
\left\|u ; H_{a, b}^{s}\right\| \leqslant c \operatorname{var} \sigma .
$$

Функция $M_{s, t}(n-2 j)$ легко вычисляется по формулам (19)-(21); здесь укажем только

$$
\begin{aligned}
& M_{1}(n-2 j)=1+(n-1) \frac{(n-2 j)^{2}}{j^{2}(n-j)^{2}}, \quad j=1,2, n-2, n-1, \\
& M_{1}(n-2 j)<\left(1-\left(\frac{n-2 j}{n-2}\right)^{2}\right)^{-1}, \quad 3 \leqslant j \leqslant n-3,
\end{aligned}
$$

и напомним, что в дивергентном случае $M_{m, m}(a)\left(M_{1}(a)\right)^{m}$.

ДокАЗАТЕЛЬСТво. По следствию 1 условие (37) обеспечивает $n-2 j \in\left(a_{*}, a^{*}\right)$. Поэтому для достаточно малого $\varepsilon>0 a, b \in\left(a_{*}, a^{*}\right)$. Применение теорем 1,6 завершает доказательство. Как и в теореме 7 , решение единственно.

Рассмотрим для оператора $\mathscr{L}$ вопрос о существовании и поведении функций типа фундаментального решения или его производных.

Теорема 9. Пусть $j \in\{1, \ldots, t\}, \quad j<n$, и выполнено (37). Тогда для произвольной точки $y \in \mathbb{R}^{n}$ и прочзвольной числовой матрицы $h$ размерности $n^{t-j} N$ система (1) с $f(x)=\operatorname{div}^{t-j} h \delta(x-y)$, где $\delta-$ дельта-функиия, имеет решение $u \in H_{\text {loc }}^{s}\left(\mathbb{R}^{n} \backslash y\right)$ такое, что

$$
\int_{R<|x-y|<2 R}\left|D^{s} u(x)\right|^{2} d x \leqslant c|h|^{2} R^{2 j-n} \quad \forall R \in(0, \infty),
$$

константа с зависит только от $K, j, n, s, t$. Такое решение единственно с точностью до полинома степени, меньшей $s$.

Отметим, что оценка (38) совпадает с оценкой для соответствуюшего решения полилапласиана $\Delta^{m}$.

ДокАЗАТЕльСтво. Для функции $f$ из условия теоремы очевидна оценка

$$
\left|I_{t} f(x)\right| \leqslant c|h| r^{j-n}
$$

$r=|x-y|, \quad I_{t}-$ потенциал Рисса (оператор с символом $|\zeta|^{-t}$ ). По следствию 1 условие (37) обеспечивает $n-2 j \in\left(a_{*}, a^{*}\right)$, поэтому найдутся числа $a, b$ такие, что $a_{*}<b<n-2 j<a<a^{*}$.

Выберем $\omega(x)=\left((r / R)^{-a}+(r / R)^{-b}\right)^{-1}, R \in(0, \infty)$ - числовой параметр. Эти веса эквивалентны при различных $R$, но не равномерно по $R \in(0, \infty)$. В силу (39) имеем $f \in H_{\omega}^{-t}$, причем

$$
\left\|f ; H_{\omega}^{-t}\right\|^{2} \leqslant c|h|^{2} R^{2 j-n} .
$$

По теореме 1 система (1) имеет единственное решение $u \in H_{\omega}^{s}$, которое удовлетворяет (7). Поскольку $\omega(x)>\frac{1}{2} \min \left\{1 ; 2^{b}\right\}>0$ в кольце $Q=\{x: R<|x-y|<2 R\}$, получаем

$$
\left\|D^{s} u ; L_{2}(Q)\right\| \leqslant c\left\|D^{s} u ; L_{2, \omega}\left(\mathbb{R}^{n}\right)\right\| \leqslant c\left\|f ; H_{\omega}^{-t}\right\| \leqslant c|h| R^{j-n / 2},
$$

что доказывает (38). 


\section{Список литературы}

1. Дубинский Ю.А. Нелинейные эллиптические и параболические уравнения // Итоги науки и техники. Современные проблемы математики. Т. 9. М.: ВИНИТИ, 1976. C. $5-130$.

2. Гаевский $X$., Грегер K., Захариас $K$. Нелинейные операторные уравнения и операторные дифференциальные уравнения. М.: Мир, 1978.

3. Дубинский Ю. А. О некоторых некоэрцитивных нелинейных уравнениях // Матем. сб. 1972. T. 87. № 3. C. 315-323.

4. De Giorgi E. Un esempio di estremali discontinue per un problema variazionale di tipo ellittico // Boll. Unione Mat. Ital. 1968. V. 1. № 1. P. 135-137.

5. Giusti E., Miranda M. Un esempio di soluzioni discontinue per un problema di minimo relativo ad un integrale regolare del calcolo delle variazioni // Boll. Unione Mat. Ital. V. 1. № 2. P. 219-226.

6. Мазья В.Г. Примеры нерегулярных решений квазилинейных эллиптических уравнений с аналитическими коэффициентами // Функцион. анализ и его прилож. 1968. Т. 2. № 3. C. $53-57$.

7. Трибель $X$. Теория интерполяции, функциональные пространства, дифференциальные операторы. М.: Мир, 1980.

8. Калита E. A. Теорема Лиувилля для эллиптических систем типа Кордеса высокого порядка // Укр. матем. журн. 1991. Т. 43. № 2. С. 199-205.

9. Калита E. A. Асимптотика решений нелинейных эллиптических систем на бесконечности // Дифференц. уравн. 1993. Т. 29. № 4. С. 627-636.

10. Калита $E$. A. Об особых точках решений нелинейных эллиптических уравнений и систем высокого порядка // Матем. сб. 1993. Т. 184. № 7. С. 117-143.

11. Cordes H. O. Über die erste Randvertaufgabe bei quasilinear Differentialgleichungen zweiter ordnung in mehr als zwei Variablen // Math. Ann. 1956. V. 131. P. 287-312.

12. Koshelev A.I., Chelkak S. I. Regularity of solutions of quasilinear elliptic systems. Leipzig: Teubner, 1985.

13. Дьнькин Е. М., Осиленкер Б. П. Весовые оценки сингулярных интегралов и их приложения // Итоги науки и техники. Матем. анализ. Т. 21. М.: ВИНИТИ, 1983. С. 42-129.

14. Берг Й., Лёфстрём Й. Интерполяционные пространства. Введение. М.: Мир, 1980.

15. Калита E. A. Точность условия Кордеса гельдеровости градиента для недивергентных эллиптических систем // Укр. матем. журн. 1995. Т. 47. № 2. С. 292-294.

16. Adams D. R. A note on Riesz potentials // Duke Math. J. 1975. V. 42. № 4. P. 765-778.

17. Hedberg L.I. On certain convolution inequalities // Proc. Amer. Math. Soc. 1972. V. 36. № 2. P. 505-510.

18. Coifman R. R., Weiss G. Extensions of Hardy spaces and their use in analysis // Bull. Amer. Math. Soc. 1977. V. 83. № 4. P. 569-645.

19. Скрыпник И. В. Методы исследования нелинейных эллиптических граничных задач. М.: Наука, 1990.

Поступило в редакцию 1.III.1995 